\title{
Search for anomaly-mediated supersymmetry breaking with the ATLAS detector based on a disappearing-track signature in $p p$ collisions at $\sqrt{s}=7 \mathrm{TeV}$
}

\author{
The ATLAS Collaboration ${ }^{\star}$ \\ CERN, 1211 Geneva 23, Switzerland
}

Received: 22 February 2012 / Revised: 6 April 2012 / Published online: 27 April 2012

(C) CERN for the benefit of the ATLAS collaboration 2012. This article is published with open access at Springerlink.com

\begin{abstract}
In models of anomaly-mediated supersymmetry breaking (AMSB), the lightest chargino is predicted to have a lifetime long enough to be detected in collider experiments. This letter explores AMSB scenarios in $p p$ collisions at $\sqrt{s}=7 \mathrm{TeV}$ by attempting to identify decaying charginos which result in tracks that appear to have few associated hits in the outer region of the tracking system. The search was based on data corresponding to an integrated luminosity of $1.02 \mathrm{fb}^{-1}$ collected with the ATLAS detector in 2011 . The $p_{\mathrm{T}}$ spectrum of candidate tracks is found to be consistent with the expectation from Standard Model background processes and constraints on the lifetime and the production cross section were obtained. In the minimal AMSB framework with $m_{3 / 2}<32 \mathrm{TeV}, m_{0}<1.5 \mathrm{TeV}, \tan \beta=5$ and $\mu>0$, a chargino having mass below $92 \mathrm{GeV}$ and a lifetime between $0.5 \mathrm{~ns}$ and $2 \mathrm{~ns}$ is excluded at $95 \%$ confidence level.
\end{abstract}

\section{Introduction}

Supersymmetry (SUSY) [1-9] is a promising solution to the hierarchy problem of the Standard Model (SM) and the search for SUSY is an important programme at the Large Hadron Collider (LHC). For each SM particle, SUSY postulates a supersymmetric partner with identical quantum numbers but with a spin that differs by $1 / 2$. Since scalar superpartners of quarks and leptons with masses equal to quarks and leptons have not been observed in previous searches, SUSY must be a broken symmetry. One mechanism which provides a calculable mass spectrum of supersymmetric particles is provided by anomaly mediation $[10,11]$. The anomaly-mediated SUSY breaking (AMSB) model provides a constrained particle mass spectrum; the ratios of the three

^e-mail: atlas.publications@cern.ch gaugino masses are given approximately as $M_{1}: M_{2}: M_{3} \approx$ 3:1: 7 where $M_{i}(i=1,2,3)$ are the bino, wino and gluino masses, respectively. The neutral wino becomes the lightest supersymmetric particle (LSP) while the charged wino becomes slightly heavier due to radiative corrections involving electroweak gauge bosons in the loops. This phenomenological feature of the nearly degenerate lightest chargino $\left(\tilde{\chi}_{1}^{ \pm}\right)$and neutralino $\left(\tilde{\chi}_{1}^{0}\right)$ has the important implication that $\tilde{\chi}_{1}^{ \pm}$predominantly decays into $\tilde{\chi}_{1}^{0}$ plus a low-momentum $(\sim 100 \mathrm{MeV}) \pi^{ \pm}$. The decay length of $\tilde{\chi}_{1}^{ \pm}$is typically expected to be a few centimeters at LHC energies; some $\tilde{\chi}_{1}^{ \pm}$ charginos could therefore decay inside the tracking volume of the ATLAS detector. The $\tilde{\chi}_{1}^{0}$ escapes detection and the softly emitted $\pi^{ \pm}$is not reconstructed. A track arising from a $\tilde{\chi}_{1}^{ \pm}$with these characteristics is classified as a disappearing track. The search described in this letter is based on this signature of decaying charginos which leads to a track having few associated hits in the outer part of the tracking volume.

\section{The ATLAS detector}

ATLAS is a multi-purpose detector [12], covering nearly the entire solid angle ${ }^{1}$ around the collision point with layers of inner tracking devices surrounded by a superconducting solenoid providing a $2 \mathrm{~T}$ magnetic field, a calorimeter system and a muon spectrometer. The inner tracking detector provides tracking in the region $|\eta|<2.5$. It consists of pixel and silicon microstrip (SCT) detectors inside a transition radiation tracker (TRT).

\footnotetext{
${ }^{1}$ ATLAS uses a right-handed coordinate system with its origin at the nominal interaction point (IP) in the centre of the detector and the $z$ axis coinciding with the axis of the beam pipe. The $x$-axis points from the IP to the centre of the LHC ring, and the $y$-axis points upward. Cylindrical coordinates $(r, \phi)$ are used in the transverse plane, $\phi$ being the azimuthal angle around the beam pipe. The pseudorapidity is defined in terms of the polar angle $\theta$ as $\eta=-\ln \tan (\theta / 2)$.
} 
Of particular importance to this analysis is the TRT which covers the region $|\eta|<2.0$. The barrel TRT is divided into inner, middle and outer concentric rings of 32 modules comprising a stack in the azimuthal angle; each covers the radial range from $563 \mathrm{~mm}$ to $1066 \mathrm{~mm}$ and $|\eta|<1.0$. A module consists of a carbon-fibre laminate shell and an array of straw tubes and has a different structure for each ring.

The calorimeter system covers the range $|\eta|<4$.9. The electromagnetic calorimeter is a lead/liquid-argon (LAr) detector in the barrel $(|\eta|<1.475)$ and endcap $(1.375<|\eta|<$ $3.2)$ regions. The hadron calorimeters are composed of a steel and scintillator barrel $(|\eta|<1.7)$, a LAr/copper endcap $(1.5<|\eta|<3.2)$ and a LAr forward system $(3.1<|\eta|<$ $4.9)$ with copper and tungsten absorbers. The muon spectrometer consists of three large superconducting toroids with 24 coils, a system of trigger chambers and precision tracking chambers which provide muon momentum measurements up to $|\eta|$ of 2.7 .

\section{Simulated event samples}

Simulated Monte Carlo (MC) events were used to assess the experimental sensitivity to given models. The minimal AMSB model is characterized by four parameters: the gravitino mass $\left(m_{3 / 2}\right)$, the universal scalar mass $\left(m_{0}\right)$, the ratio of Higgs vacuum expectation values at the electroweak scale $(\tan \beta)$ and the sign of the higgsino mass term $(\operatorname{sgn}(\mu))$. In this letter, ISASUSY from ISAJET v7.80 [13] was used to calculate the SUSY mass spectrum and the decay tables. The MC samples were produced using HERWIG++ [14] with MRST2007 LO* [15] parton distribution functions. These samples were produced using the parameter tune described in [16] and a detector simulation based on GEANT4 [17, 18] with multiple $p p$ interactions per event (pile-up) to match what was observed in data. Given the chargino mass $\left(m_{\tilde{\chi}_{1}^{ \pm}}\right)$ limit by the LEP2 searches [19-21] of $m_{\tilde{\chi}_{1}^{ \pm}} \simeq 92 \mathrm{GeV}$ at $95 \%$ confidence level (CL), the signal models shown in Table 1 were tested. A large value of $m_{0}$ was used in order to prevent the existence of a tachyonic slepton; this also assigns heavy masses to the squarks and sleptons, thereby avoiding constraints from flavour-changing neutral current and CPviolation measurements. In this search, the production processes $\tilde{g} \tilde{g}, \tilde{q} \tilde{g}$ and $\tilde{q} \tilde{q}$ were considered. The signal samples were normalized using next-to-leading-order (NLO) cross sections determined with PROSPINO [22]. The chargino lifetime $\left(\tau_{\tilde{\chi}_{1}^{ \pm}}\right)$was set to $1 \mathrm{~ns}$, the value for which this analysis has the highest sensitivity. The branching fraction for the decay $\tilde{\chi}_{1}^{ \pm} \rightarrow \tilde{\chi}_{1}^{0} \pi^{ \pm}$was set to $100 \%$. Samples with different lifetime values for each signal model were derived by applying event weights so that the distribution of the proper lifetime follows that for a given lifetime value.
Table 1 Summary of AMSB signal parameters, chargino masses and their NLO cross sections with $\tan \beta=5$ and $\operatorname{sgn}(\mu)=+1$

\begin{tabular}{lllcl}
\hline Signal & $m_{0}[\mathrm{TeV}]$ & $m_{3 / 2}[\mathrm{TeV}]$ & $m_{\tilde{\chi}_{1}^{ \pm}}[\mathrm{GeV}]$ & Cross section $[\mathrm{pb}]$ \\
\hline LL01 & 1.5 & 32 & 90.2 & $6.10 \times 10^{-2}$ \\
LL02 & 1.8 & 41 & 117.8 & $7.65 \times 10^{-3}$ \\
LL03 & 2.0 & 51 & 147.7 & $1.00 \times 10^{-3}$ \\
\hline
\end{tabular}

In the model, gluinos and squarks are expected to be produced copiously via the strong interaction in $p p$ collisions. The decay cascade of these to the $\tilde{\chi}_{1}^{ \pm}$and $\tilde{\chi}_{1}^{0}$ produces multiple jets with high transverse momentum $\left(p_{\mathrm{T}}\right)$. LSPs escape from the detector, resulting in an event topology with multiple jets and large missing transverse momentum $\left(E_{\mathrm{T}}^{\text {miss }}\right)$. Chargino tracks are expected to have significant transverse momentum since the difference between the gluino and chargino masses is large; the chargino track typically has $p_{\mathrm{T}}>50 \mathrm{GeV}$ and is well isolated from the jet activity in the event.

\section{Data and event selection}

The analysis was based on $p p$ collision data at $\sqrt{s}=7 \mathrm{TeV}$ recorded from March to July 2011. The corresponding integrated luminosity, after the application of beam, detector and data quality requirements, was $1.02 \mathrm{fb}^{-1}$. Events were selected at the trigger level by requiring at least one jet with a transverse momentum, measured at the electromagnetic scale, above $75 \mathrm{GeV}$, and a missing transverse momentum above $55 \mathrm{GeV}$.

Jets were reconstructed using the anti- $k_{t}$ algorithm [23] with a distance parameter of 0.4 . The inputs to the jet reconstruction algorithm were three-dimensional topological calorimeter energy clusters [24]. The measurement of jet transverse momentum at the electromagnetic scale $\left(p_{\mathrm{T}}^{\mathrm{jet}, \mathrm{EM}}\right)$ underestimates the true momentum due to the nature of the non-compensating calorimeters and the dead material. Thus, an average correction [25], depending on $\eta$ and $p_{\mathrm{T}}^{\text {jet,EM }}$, was applied to obtain the calibrated jet $p_{\mathrm{T}}$. Jets with $p_{\mathrm{T}}>20 \mathrm{GeV}$ and $|\eta|<3.2$ were selected. Electron candidates were selected with "medium" purity cuts, as described in Ref. [26]. Furthermore, electrons were required to fulfill the requirements of $p_{\mathrm{T}}>10 \mathrm{GeV},|\eta|<2.47$ and $\sum_{\Delta R<0.2} p_{\mathrm{T}}^{\text {track }} / p_{\mathrm{T}}<0.1$, where $\sum_{\Delta R<0.2} p_{\mathrm{T}}^{\text {track }}$ is the sum of $p_{\mathrm{T}}$ for all the tracks with $p_{\mathrm{T}}>1 \mathrm{GeV}$ in a cone of $\Delta R \equiv \sqrt{(\Delta \eta)^{2}+(\Delta \phi)^{2}}<0.2$ around the electron candidate, excluding the $p_{\mathrm{T}}$ of the electron candidate itself. Muon candidates were identified by an algorithm which combines a track reconstructed in the muon spectrometer with a track in the inner detector. Furthermore, muons were required to have $p_{\mathrm{T}}>10 \mathrm{GeV}$ and $|\eta|<2.7$, and to be isolated [27]: 
the sum of $p_{\mathrm{T}}$ of tracks within a cone of $\Delta R<0.2$ around the muon candidate (excluding the muon candidate itself) was required to be less than $1.8 \mathrm{GeV}$.

Following the object reconstruction described above, overlaps between jets and leptons were resolved. First, any jet candidate lying within a distance of $\Delta R<0.2$ of an electron was discarded. Then, any lepton candidates within a distance of $\Delta R<0.4$ of any surviving jet were discarded.

The calculation of $E_{\mathrm{T}}^{\text {miss }}$ was based on the transverse momenta of jets and lepton candidates, and all clusters in the calorimeter that are not associated to such objects [28].

In order to suppress non-collision background events, additional selection criteria [25] were applied to jets. Signal candidate events were required to have no electron or muon candidates (lepton veto), $E_{\mathrm{T}}^{\text {miss }}>130 \mathrm{GeV}$ and three leading (highest $p_{\mathrm{T}}$ ) jets with $p_{\mathrm{T}}>130 \mathrm{GeV}$ for one jet and $p_{\mathrm{T}}>60 \mathrm{GeV}$ for another two jets ("kinematic selection"). The trigger selection is fully efficient for signal events satisfying the kinematic selection requirements.

The search described in this letter was based on the detection of charginos decaying in the TRT. The average number of hits on a track going through the TRT in the central region is about 34 and consecutive hits can be observed along the track with small radial spacing between adjacent hits. This feature provides the capability of substantial discrimination between penetrating and decaying charged particles. If a chargino decays in the volume of the inner or middle TRT modules, multiple hits associated to the chargino track are expected in the SCT detector but not in the outer TRT subdetector. Such a chargino track candidate can be fully reconstructed by the ATLAS standard track reconstruction algorithm.

The chargino candidate tracks were required to fulfill the following criteria:

(1) The track should have at least one hit in the innermost layer of the pixel detector.

(2) The track should have at least six hits in the SCT.

(3) The track should have $\left|d_{0}\right|<1.5 \mathrm{~mm}$ and $\left|z_{0} \sin \theta\right|<$ $1.5 \mathrm{~mm}$, where $d_{0}$ and $z_{0}$ are the transverse and longitudinal impact parameters.

(4) There should be no other tracks with $p_{\mathrm{T}}>0.5 \mathrm{GeV}$ within a cone of radius $\Delta R=0.05$.

(5) The candidate track should have the highest $p_{\mathrm{T}}$ among the isolated tracks in the event and have $p_{\mathrm{T}}$ above $10 \mathrm{GeV}$.

(6) The track should point to the TRT barrel layers and not point to the inactive regions around $\eta=0$.

(7) The number of hits in the TRT outer module associated to the track ( $\left.N_{\mathrm{TRT}}^{\text {outer }}\right)$ should be fewer than five.

The first four criteria were applied to all tracks in the event in order to ensure a well-reconstructed primary track whereas

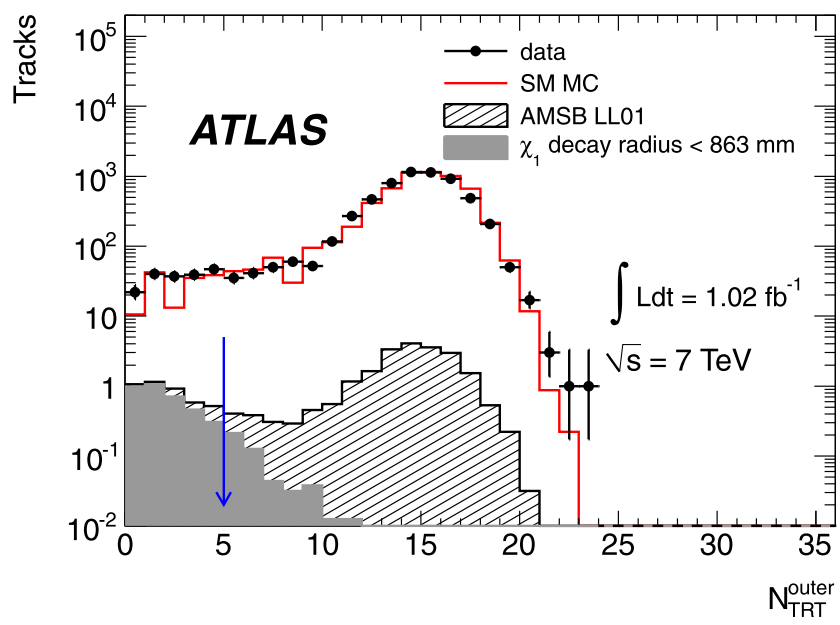

Fig. 1 The $N_{\text {TRT }}^{\text {outer }}$ distribution for data and signal events (LL01, $\left.\tau_{\tilde{\chi}_{1}^{ \pm}}=1 \mathrm{~ns}\right)$ with the high- $p_{\mathrm{T}}$ isolated track selection. The selection boundary is indicated by the arrow. The expectation from SM MC events, normalized to the number of observed events, is also shown. When charginos decay before reaching the TRT outer module, $N_{\text {TRT }}^{\text {outer }}$ is expected to have a value near zero; conversely, SM charged particles traversing the TRT typically have $N_{\mathrm{TRT}}^{\text {outer }} \simeq 15$

the fifth is meant to select chargino tracks that usually have the highest $p_{\mathrm{T}}$ in the event. The chargino tracks sufficiently fulfill the fifth criterion. The sixth criterion was based on the extrapolated track position, and was set to avoid inactive regions of the TRT. This requirement helped to reject fake disappearing tracks and works as an effective acceptance cut of $|\eta|<0.63$. For the seventh criterion, $N_{\mathrm{TRT}}^{\text {outer }}$ was calculated by counting TRT hits lying on the extrapolated track. The hits satisfying $d<r_{\text {straw }}$ were taken into account, where $d$ is the distance between the hit and the track in the transverse plane and $r_{\text {straw }}$ is the radius of the straw tube. Hereafter, unless explicitly stated otherwise, "high$p_{\mathrm{T}}$ isolated track selection" and "disappearing track selection" indicate criteria (1)-(6) and (1)-(7), respectively. Figure 1 shows the $N_{\mathrm{TRT}}^{\text {outer }}$ distributions with the high- $p_{\mathrm{T}}$ isolated track selection requirements for data, signal and SM MC events. When charginos decay before reaching the TRT outer module, $N_{\text {TRT }}^{\text {outer }}$ is expected to have a value near zero; conversely, SM charged particles traversing the TRT typically have $N_{\mathrm{TRT}}^{\text {outer }} \simeq 15$. The sample of selected tracks after requirements (1)-(6) is dominated by through-going tracks with $N_{\mathrm{TRT}}^{\text {outer }} \simeq 15$. Criterion (7) removes the vast majority of these tracks: although it reduces the signal efficiency, it enhances the expected signal to background ratio very strongly. These criteria select charginos decaying in the region $514<r<863 \mathrm{~mm}$ effectively. The data reduction is summarized in Table 2. After the application of all kinematic and track selection criteria, 185 candidate events remained. 
Table 2 Summary of selection cuts and data reduction. The selection efficiencies for each AMSB signal model are also shown

\begin{tabular}{lrrrr}
\hline Selection & \multirow{2}{*}{ Data } & \multicolumn{3}{l}{ Signal efficiency [\%] } \\
\cline { 3 - 5 } & & LL01 & LL02 & LL03 \\
\hline Trigger selection and non-collision rejection & 1491012 & 90.2 & 90.2 & 89.3 \\
$e / \mu$ veto & 1390171 & 77.1 & 75.2 & 73.7 \\
$E_{\mathrm{T}}^{\text {miss }}>130 \mathrm{GeV}$ & 80971 & 67.9 & 68.8 & 69.4 \\
Jet $p_{\mathrm{T}}$ requirements & 18345 & 66.5 & 68.1 & 68.8 \\
High- $p_{\mathrm{T}}$ isolated track & 6042 & 40.8 & 42.9 & 43.5 \\
Disappearing track & 185 & 6.8 & 7.5 & 7.4 \\
\hline
\end{tabular}

\section{Background estimation}

With the selection criteria described above, there are two main background sources for high- $p_{\mathrm{T}}$ disappearing tracks:

- Charged hadrons (mostly charged pions) interacting with material in the TRT detector.

- Low- $p_{\mathrm{T}}$ charged particles whose $p_{\mathrm{T}}$ is badly measured due to scattering in the inner detector material.

The two categories are labelled as "high- $p_{\mathrm{T}}$ interacting hadron track" and "bad track" backgrounds, respectively. Figure 2 shows schematically the origins of disappearing high- $p_{\mathrm{T}}$ tracks. According to the MC simulation, high- $p_{\mathrm{T}}$ interacting hadron tracks were responsible for more than $95 \%$ of the background tracks. Electrons having low $p_{\mathrm{T}}$ can be classified as disappearing tracks due to bremsstrahlung, however, the contribution of these tracks was negligibly small after the lepton veto and the track selection criterion (5).

The fraction of events containing these background tracks is expected to be $\sim 10^{-4}$; background estimation based on the MC simulation would therefore suffer from large uncertainties due to the lack of sufficient MC statistics and also from the difficulty in simulating the properties of these background mechanisms. A data-driven background estimation technique was therefore used to estimate the background track $p_{\mathrm{T}}$ spectrum, which used control samples enriched in the two background categories. The main contribution to the high- $p_{\mathrm{T}}$ interacting hadron background originated from charged hadrons in jets and $\tau$ hadronic decays. In the $p_{\mathrm{T}}$ range above $10 \mathrm{GeV}$, where inelastic interactions dominate, the interaction rate has nearly no $p_{\mathrm{T}}-$ dependence [29]. Therefore, the $p_{\mathrm{T}}$ spectrum of interacting hadron tracks was obtained from that of non-interacting hadron tracks. By adopting the same kinematic selection criteria as those for the signal and ensuring penetration through the TRT detector by requiring $N_{\text {TRT }}^{\text {outer }}>10$, a pure sample of high- $p_{\mathrm{T}}$ non-interacting hadron tracks was obtained. The contamination from bad tracks and any chargino signal was removed by requiring the calorimeter activity associated to the track, $\sum_{\Delta R<0.1} E_{\mathrm{T}}^{\text {clus }} / p_{\mathrm{T}}^{\text {track }}$, to be larger than 0.3 , where $p_{\mathrm{T}}^{\text {track }}$ is the $p_{\mathrm{T}}$ of the track and $\sum_{\Delta R<0.1} E_{\mathrm{T}}^{\text {clus }}$ is the

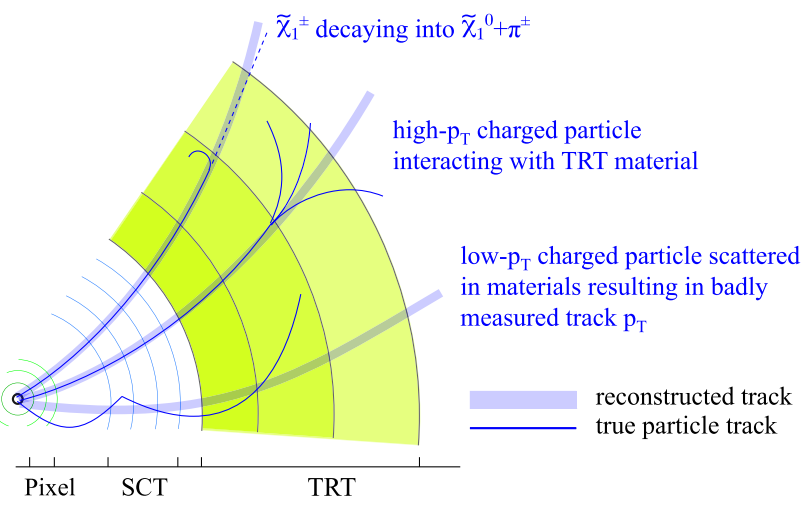

Fig. 2 Origins of disappearing high- $p_{\mathrm{T}}$ tracks

sum of cluster transverse energies in a cone of $\Delta R=0.1$ around the track. Simulation studies indicated that the $p_{\mathrm{T}}$ spectrum of bad tracks depends little on the production process. A sample with an enhanced bad track contribution was therefore obtained with the same track quality requirements as for the chargino track, but requiring $E_{\mathrm{T}}^{\text {miss }}<100 \mathrm{GeV}$. The $E_{\mathrm{T}}^{\text {miss }}$ requirement makes this sample orthogonal to the signal search sample. In addition, the number of pixel hits associated to the track was required to be zero, and $\sum_{\Delta R<0.1} E_{\mathrm{T}}^{\text {clus }} / p_{\mathrm{T}}^{\text {track }}<0.3$ in order to reject possible contributions from high- $p_{\mathrm{T}}$ interacting hadron tracks and to enhance the purity of bad tracks. The requirement on the number of pixel hits had negligible impact on the shape of the reconstructed $p_{\mathrm{T}}$ spectrum. The purity of bad tracks was close to $100 \%$ after these requirements.

An ansatz functional form $(1+x)^{a_{0}} / x^{a_{1}+a_{2} \ln (x)}$ was fitted to the $p_{\mathrm{T}}$ spectrum of the control sample of the high$p_{\mathrm{T}}$ non-interacting hadron tracks, where $x \equiv p_{\mathrm{T}}^{\text {track }}$ and $a_{i}$ $(i=0,1,2)$ are fit parameters. Figure 3(a) shows the track $p_{\mathrm{T}}$ distribution and the shape derived from a maximum likelihood fit. Alternative fit functions gave shapes that agreed with each other and with the original form within the fit uncertainties. The choice of functional form in this analysis was based on the $\chi^{2}$ values.

Bad tracks could have anomalously high values of $p_{\mathrm{T}}$ and become a significant background. Therefore, for the bad track background shape, a flat term representing the high- $p_{\mathrm{T}}$ tail was added to give an estimate in the region of interest. 


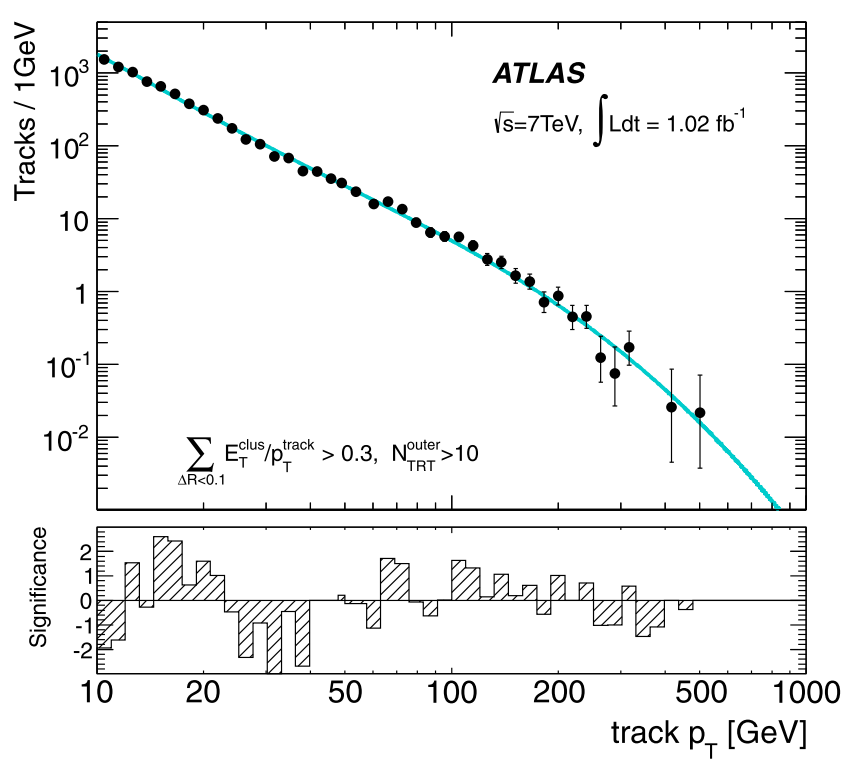

(a) High- $p_{\mathrm{T}}$ hadron track sample

Fig. 3 The $p_{\mathrm{T}}$ distributions of high- $p_{\mathrm{T}}$ hadron track (a) and bad track (b) background control samples. The data and the fitted model are shown by the solid circles and the line, respectively. The significance

The resulting functional form was $(1+x)^{b_{0}} / x^{b_{1}+b_{2} \ln (x)}+$ $b_{3}$, where $b_{i}(i=0,1,2,3)$ are fit parameters. The shape of the bad track background is shown in Fig. 3(b).

\section{Signal extraction and constraints on the AMSB chargino}

In order to evaluate how well the observed data agree with a given signal model, a statistical test was performed based on a maximum likelihood. The likelihood function for the sample of observed events $\left(n_{\mathrm{obs}}\right)$, using the track $p_{\mathrm{T}}$, is defined as:

$\prod^{n_{\text {obs }}} \frac{\mu_{\mathrm{s}} n_{\mathrm{s}}^{\exp } \mathscr{L}_{\mathrm{s}}+n_{\mathrm{b}}\left\{\left(1-f_{\text {bad }}\right) \mathscr{L}_{\text {had }}+f_{\text {bad }} \mathscr{L}_{\text {bad }}\right\}}{n_{\mathrm{b}}+\mu_{\mathrm{s}} n_{\mathrm{s}}^{\exp }}$,

where $\mu_{\mathrm{s}}, n_{\mathrm{s}}^{\exp }, n_{\mathrm{b}}$ and $f_{\mathrm{bad}}$ are the signal strength (i.e. the ratio of a given cross section to its predicted value), the expected number of signal events for a given model, the number of background events and the fraction of bad tracks in the background, respectively. The parameters $\mu_{\mathrm{s}}, n_{\mathrm{b}}$ and $f_{\mathrm{bad}}$ were left free in the fit. The probability density functions of signal, interacting hadron track and bad track, $\mathscr{L}_{\mathrm{s}}, \mathscr{L}_{\text {had }}$ and $\mathscr{L}_{\text {bad }}$, are shown in Fig. 4. The full shape of the distributions for $p_{\mathrm{T}}>10 \mathrm{GeV}$ was fitted with the two background contributions, and a signal contribution was also included in the fit for $p_{\mathrm{T}}>50 \mathrm{GeV}$. A small signal contribution below $p_{\mathrm{T}}=50 \mathrm{GeV}$ was neglected. The effects of systematic uncertainties were incorporated via constraint terms on

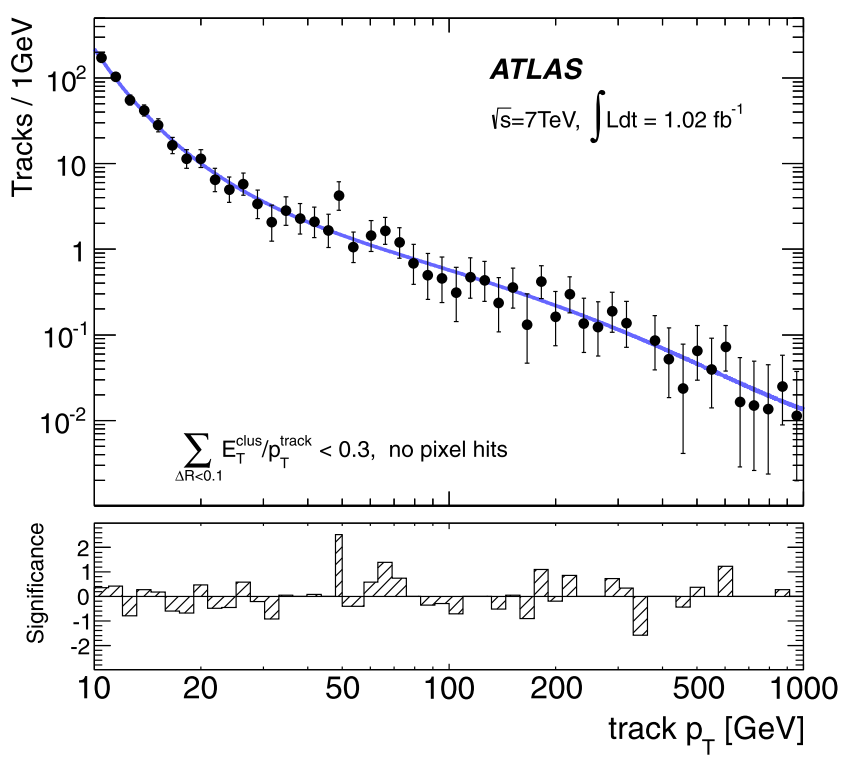

(b) Bad track sample

of the data-model difference on a bin-by-bin basis is also shown at the bottom of each figure

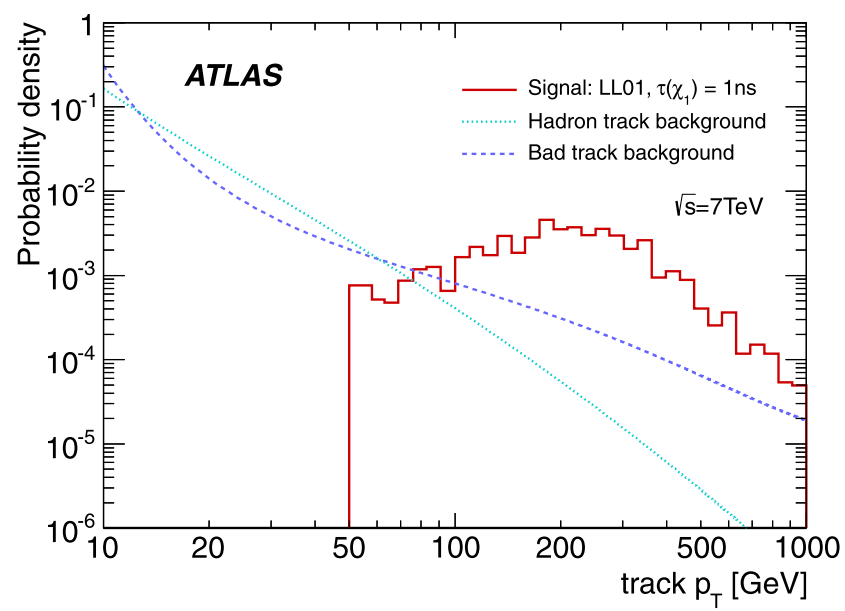

Fig. 4 Probability densities of the signal (LL01, $\tau_{\tilde{\chi}_{1}^{ \pm}}=1 \mathrm{~ns}$ ) and background components, shown as a function of track $p_{\mathrm{T}}$. In the signal case, only the region $p_{\mathrm{T}}>50 \mathrm{GeV}$ is shown

nuisance parameters. The overall normalisation of the signal and the parameters describing the background track $p_{\mathrm{T}}$ shapes were set as nuisance parameters; they were treated with a normal distribution and multivariate normal distributions with covariance matrices obtained by the fit of the background control samples, respectively.

A total uncertainty of $\pm 25 \%$ was found for the signal normalisation; the main contribution comes from the uncertainties in the theoretical cross section from the renormalisation and factorisation scales $( \pm 18 \%)$ and the parton distribution functions $( \pm 9 \%)$. The jet energy scale [25], the 


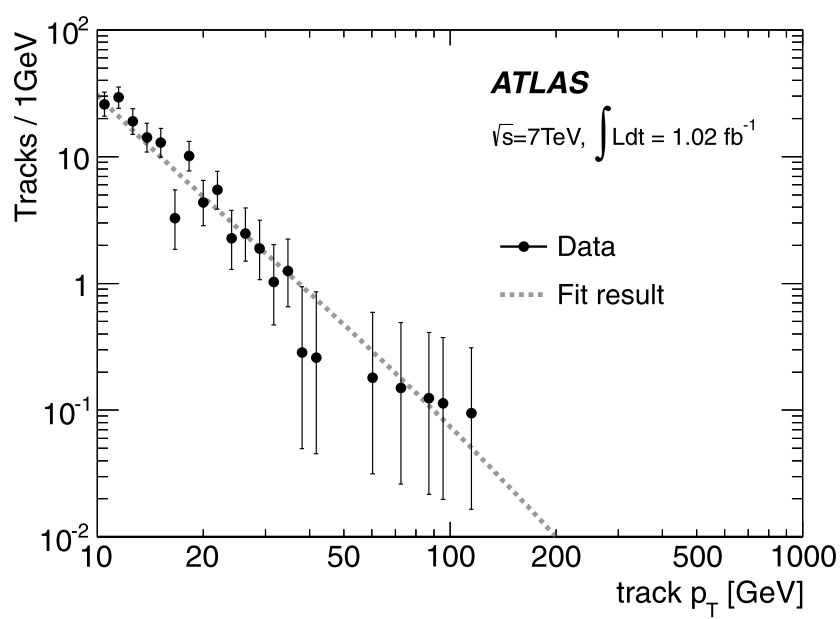

Fig. 5 The $p_{\mathrm{T}}$ distribution of candidate tracks with the best-fit shape of the "signal + background" model. The signal point of LL01 and $\tau_{\tilde{\chi}_{1}^{ \pm}}=1 \mathrm{~ns}$ are used, but the best-fit signal contribution was found to be zero

track reconstruction efficiency [30] and the integrated luminosity $[31,32]$ could alter the signal yield; their contributions were estimated to be $\pm 9 \%, \pm 2 \%$ and $\pm 3.7 \%$, respectively. The systematic uncertainties due to pile-up were evaluated by examining the stability of the signal acceptance and the $p_{\mathrm{T}}$ spectra of background tracks as a function of the number of $p p$ interactions. Both data and signal MC were used for this purpose, and the resulting uncertainties were found to be negligible.

Figure 5 shows the best-fit shape of the "signal + background" model for the sample signal point LL01 with $\tau_{\tilde{\chi}_{1}^{ \pm}}=$ $1 \mathrm{~ns}\left(n_{\mathrm{S}}^{\exp }=4.2\right)$. The fit resulted in $n_{\mathrm{b}}=185 \pm 14$ and the best fit values of $\mu_{\mathrm{s}}$ and $f_{\text {bad }}$ were zero; upper limits of $\mu_{\mathrm{s}}<0.15$ and $f_{\text {bad }}<4.0 \times 10^{-2}$ were set at $68 \% \mathrm{CL}$. The $p$-value for the consistency of the observed data with the background-only hypothesis was calculated to be 0.5 , showing that the observed track $p_{\mathrm{T}}$ spectrum was in agreement with the background expectation. The result also indicated that interacting hadron tracks were the dominant background, consistent with $\mathrm{MC}$ predictions.

The expected background and observed events in the region $p_{\mathrm{T}}>50 \mathrm{GeV}$ were $13 \pm 1$ and 5 , respectively; this background estimate was derived from the background-only fit in the region $10<p_{\mathrm{T}} \leq 50 \mathrm{GeV}$. Model-independent upper limits were set on the cross section times acceptance for non-SM processes with the final state satisfying the kinematic and track selection criteria. Figure 6 shows $95 \%$ CL upper limits on the cross section times acceptance for candidate tracks with $p_{\mathrm{T}}>p_{\mathrm{T}}^{0}$ as a function of $p_{\mathrm{T}}^{0}$. The $95 \%$ CL upper limit on the cross section for a given model was set by the point where the CL of the signal + background hypothesis based on the profile likelihood ratio [33] and

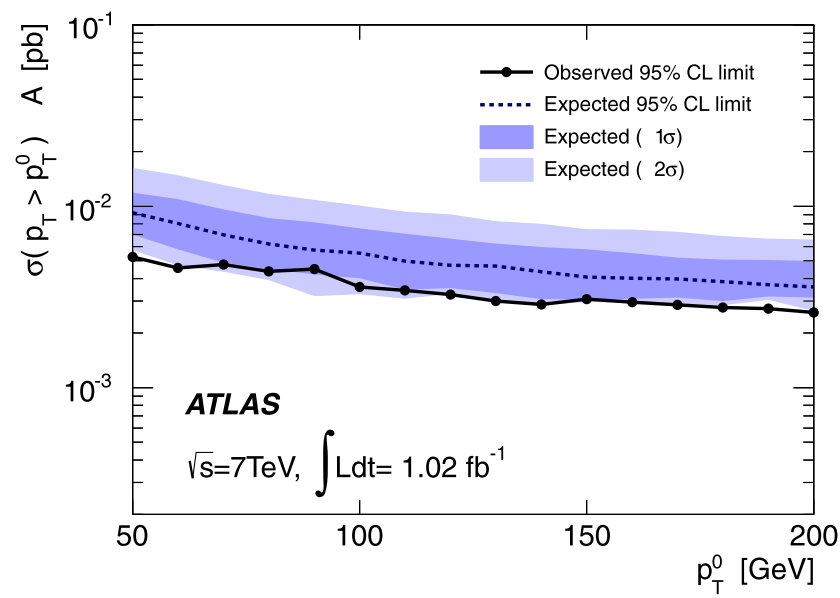

Fig. 6 Model-independent upper limits on the cross section $(\sigma)$ times acceptance $(A)$ for a non-SM physics process containing an isolated, disappearing track with $p_{\mathrm{T}}>p_{\mathrm{T}}^{0}$ as a function of $p_{\mathrm{T}}^{0}$. The observed and expected bounds at $95 \% \mathrm{CL}$ are shown

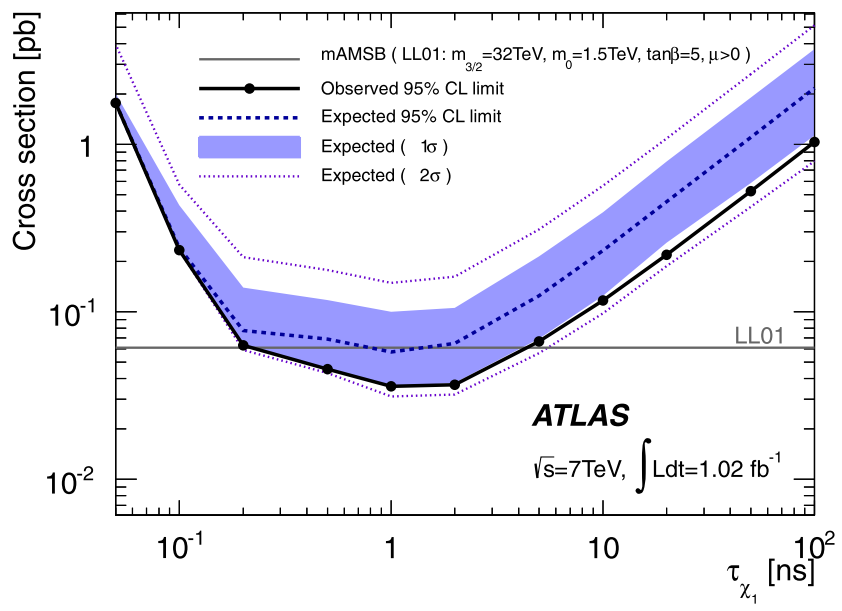

Fig. 7 The observed and expected $95 \%$ CL upper limits on the signal cross section as a function of chargino lifetime for $m_{\tilde{\chi}_{1}^{ \pm}}=90.2 \mathrm{GeV}$. The band and the dotted line indicate the range in which the limit is expected to lie due to the fluctuations in the expected background

the $C L s$ method $[34,35]$ falls below $5 \%$ when scanning the $\mathrm{CL}$ along various values of $\mu_{\mathrm{s}}$. Figure 7 shows the observed limit on the signal cross section at $95 \% \mathrm{CL}$ as a function of $\tau_{\tilde{\chi}_{1}^{ \pm}}$for the signal model LL01. Limits on the chargino lifetime were also set: $\tau_{\tilde{\chi}_{1}^{ \pm}}<0.2$ or $\tau_{\tilde{\chi}_{1}^{ \pm}}>4 \mathrm{~ns}$ for a chargino with a mass of $90 \mathrm{GeV}$. Moreover, a constraint on the chargino mass and lifetime was set by the scan of the observed cross section limits for the benchmark models, as shown in Fig. 8. In the framework of minimal AMSB with $m_{3 / 2}<32 \mathrm{TeV}, m_{0}<1.5 \mathrm{TeV}, \tan \beta=5$ and $\mu>0$, a chargino with $m_{\tilde{\chi}_{1}^{ \pm}}<92 \mathrm{GeV}$ and $0.5<\tau_{\tilde{\chi}_{1}^{ \pm}}<2$ ns was excluded at $95 \% \mathrm{CL}$. 


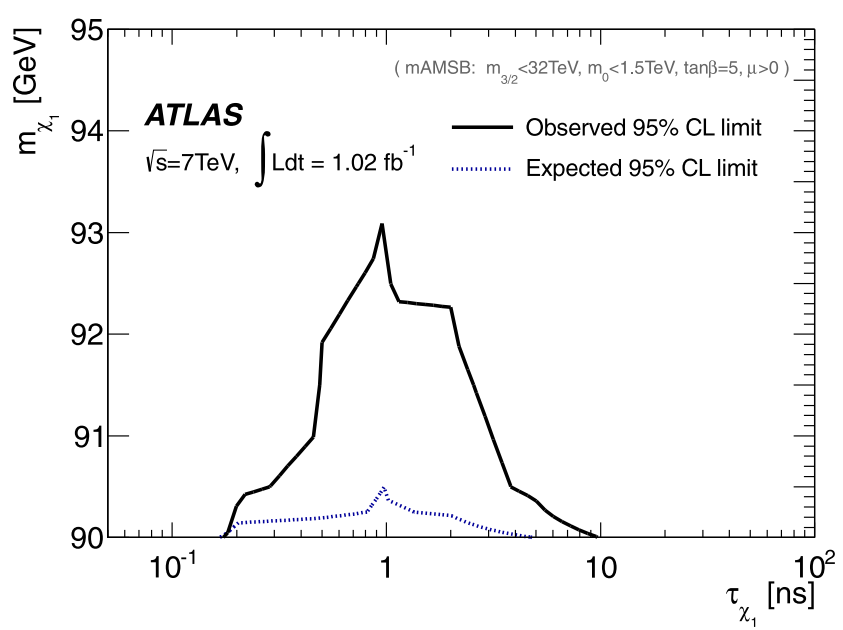

Fig. 8 The constraint on the chargino mass and lifetime in a minimal AMSB model with $m_{3 / 2}<32 \mathrm{TeV}, m_{0}<1.5 \mathrm{TeV}, \tan \beta=5$ and $\mu>0$. The observed and expected bounds at $95 \% \mathrm{CL}$ are shown

\section{Conclusion}

The results of a search for long-lived charginos in $p p$ collisions with the ATLAS detector using $1.02 \mathrm{fb}^{-1}$ of data were presented in the context of AMSB scenarios. The analysis used a signature of high- $p_{\mathrm{T}}$ isolated tracks with few associated hits in the outer part of the ATLAS tracking system. The $p_{\mathrm{T}}$ spectrum of observed candidate tracks was found to be consistent with the expectation from SM background processes. Constraints on the AMSB chargino mass and lifetime were set; a chargino having $m_{\tilde{\chi}_{1}^{ \pm}}<92 \mathrm{GeV}$ and $0.5<\tau_{\tilde{\chi}_{1}^{ \pm}}<2$ ns was excluded at $95 \% \mathrm{CL}$.

Acknowledgements We thank CERN for the very successful operation of the LHC, as well as the support staff from our institutions without whom ATLAS could not be operated efficiently.

We acknowledge the support of ANPCyT, Argentina; YerPhI, Armenia; ARC, Australia; BMWF, Austria; ANAS, Azerbaijan; SSTC, Belarus; CNPq and FAPESP, Brazil; NSERC, NRC and CFI, Canada; CERN; CONICYT, Chile; CAS, MOST and NSFC, China; COLCIENCIAS, Colombia; MSMT CR, MPO CR and VSC CR, Czech Republic; DNRF, DNSRC and Lundbeck Foundation, Denmark; EPLANET and ERC, European Union; IN2P3-CNRS, CEA-DSM/IRFU, France; GNAS, Georgia; BMBF, DFG, HGF, MPG and AvH Foundation, Germany; GSRT, Greece; ISF, MINERVA, GIF, DIP and Benoziyo Center, Israel; INFN, Italy; MEXT and JSPS, Japan; CNRST, Morocco; FOM and NWO, Netherlands; RCN, Norway; MNiSW, Poland; GRICES and FCT, Portugal; MERYS (MECTS), Romania; MES of Russia and ROSATOM, Russian Federation; JINR; MSTD, Serbia; MSSR, Slovakia; ARRS and MVZT, Slovenia; DST/NRF, South Africa; MICINN, Spain; SRC and Wallenberg Foundation, Sweden; SER, SNSF and Cantons of Bern and Geneva, Switzerland; NSC, Taiwan; TAEK, Turkey; STFC, the Royal Society and Leverhulme Trust, United Kingdom; DOE and NSF, United States of America.
The crucial computing support from all WLCG partners is acknowledged gratefully, in particular from CERN and the ATLAS Tier-1 facilities at TRIUMF (Canada), NDGF (Denmark, Norway, Sweden), CC-IN2P3 (France), KIT/GridKA (Germany), INFN-CNAF (Italy), NL-T1 (Netherlands), PIC (Spain), ASGC (Taiwan), RAL (UK) and BNL (USA) and in the Tier-2 facilities worldwide.

Open Access This article is distributed under the terms of the Creative Commons Attribution License which permits any use, distribution, and reproduction in any medium, provided the original author(s) and the source are credited.

\section{References}

1. Y.A. Golfand, E.P. Likhtman, JETP Lett. 13, 323 (1971)

2. A. Neveu, J.H. Schwarz, Nucl. Phys. B 31, 86 (1971)

3. A. Neveu, J.H. Schwarz, Phys. Rev. D 4, 1109 (1971)

4. P. Ramond, Phys. Rev. D 3, 2415 (1971)

5. D.V. Volkov, V.P. Akulov, Phys. Lett. B 46, 109 (1973)

6. J. Wess, B. Zumino, Phys. Lett. B 49, 52 (1974)

7. J. Wess, B. Zumino, Nucl. Phys. B 70, 39 (1974)

8. P. Fayet, Phys. Lett. B 69, 489 (1977)

9. G.R. Farrar, P. Fayet, Phys. Lett. B 76, 575 (1978)

10. L. Randall, R. Sundrum, Nucl. Phys. B 557, 79 (1999)

11. G.F. Giudice, M.A. Luty, H. Murayama, R. Rattazzi, JHEP 12, 027 (1998)

12. The ATLAS Collaboration, JINST 3, S08003 (2008)

13. F.E. Paige, S.D. Protopopescu, H. Baer, X. Tata, arXiv:hep-ph/ 0312045 (2003)

14. M. Bahr et al., Eur. Phys. J. C 58, 639 (2008)

15. A. Sherstnev, R.S. Thorne, Eur. Phys. J. C 55, 553 (2008)

16. The ATLAS Collaboration, ATLAS Note ATLAS-CONF-2010031, http://cdsweb.cern.ch/record/1277665

17. S. Agostinelli et al., Nucl. Instrum. Meth. A 506, 250 (2003)

18. The ATLAS Collaboration, Eur. Phys. J. C 70, 823 (2010)

19. A. Heister et al. (The ALEPH Collaboration) Phys. Lett. B 533, 223 (2002)

20. G. Abbiendi et al. (The OPAL Collaboration) Eur. Phys. J. C 29, 479 (2003)

21. J. Abdallah et al. (The DELPHI Collaboration) Eur. Phys. J. C 34, 145 (2004)

22. W. Beenakker, R. Hopker, M. Spira, P.M. Zerwas, Nucl. Phys. B 492, 51 (1997)

23. M. Cacciari, G.P. Salam, G. Soyez, JHEP 04, 063 (2008)

24. W. Lampl et al., ATLAS Note ATL-LARG-PUB-2008-002, http://cdsweb.cern.ch/record/1099735

25. The ATLAS Collaboration, arXiv:1112.6426 (2011), submitted to Eur. Phys. J. C

26. The ATLAS Collaboration, JHEP 12, 060 (2010)

27. The ATLAS Collaboration, Phys. Rev. D 85, 012006 (2011)

28. The ATLAS Collaboration, Eur. Phys. J. C 72, 1844 (2012)

29. K. Nakamura et al., J. Phys. G 37, 075021 (2010), corresponding data may be found at pp. 398-399

30. The ATLAS Collaboration, New J. Phys. 13, 053033 (2011)

31. The ATLAS Collaboration, ATLAS Note ATLAS-CONF-2011116, http://cdsweb.cern.ch/record/1376384

32. The ATLAS Collaboration, Eur. Phys. J. C 71, 1630 (2011)

33. G. Cowan, K. Cranmer, E. Gross, O. Vitells, Eur. Phys. J. C 71, $1554(2011)$

34. T. Junk, Nucl. Instrum. Meth. A 434, 435 (1999)

35. A.L. Read, J. Phys. G 28, 2693 (2002) 


\section{The ATLAS Collaboration}

G. Aad $^{48}$, B. Abbott ${ }^{111}$, J. Abdallah ${ }^{11}$, A.A. Abdelalim ${ }^{49}$, A. Abdesselam ${ }^{118}$, O. Abdinov ${ }^{10}$, B. Abi ${ }^{112}$, M. Abolins ${ }^{88}$, H. Abramowicz ${ }^{153}$, H. Abreu ${ }^{115}$, E. Acerbi ${ }^{89 a, 89 b}$, B.S. Acharya ${ }^{164 a, 164 b}$, D.L. Adams ${ }^{24}$, T.N. Addy ${ }^{56}$, J. Adelman ${ }^{175}$, M. Aderholz ${ }^{99}$, S. Adomeit ${ }^{98}$, P. Adragna ${ }^{75}$, T. Adye ${ }^{129}$, S. Aefsky ${ }^{22}$, J.A. Aguilar-Saavedra ${ }^{124 b, a}$, M. Aharrouche ${ }^{81}$, S.P. Ahlen ${ }^{21}$, F. Ahles ${ }^{48}$, A. Ahmad ${ }^{148}$, M. Ahsan ${ }^{40}$, G. Aielli ${ }^{133 a, 133 b}$, T. Akdogan ${ }^{18 a}$, T.P.A. Åkesson ${ }^{79}$, G. Akimoto ${ }^{155}$, A.V. Akimov ${ }^{94}$, A. Akiyama ${ }^{67}$, M.S. Alam ${ }^{1}$, M.A. Alam ${ }^{76}$, J. Albert ${ }^{169}$, S. Albrand ${ }^{55}$, M. Aleksa ${ }^{29}$, I.N. Aleksandrov $^{65}$, F. Alessandria ${ }^{89 a}$, C. Alexa ${ }^{25 a}$, G. Alexander ${ }^{153}$, G. Alexandre ${ }^{49}$, T. Alexopoulos ${ }^{9}$, M. Alhroob ${ }^{20}$, M. Aliev $^{15}$, G. Alimonti ${ }^{89 a}$, J. Alison ${ }^{120}$, M. Aliyev ${ }^{10}$, P.P. Allport ${ }^{73}$, S.E. Allwood-Spiers ${ }^{53}$, J. Almond ${ }^{82}$, A. Aloisio ${ }^{102 a, 102 b}$, R. Alon ${ }^{171}$, A. Alonso ${ }^{79}$, B. Alvarez Gonzalez ${ }^{88}$, M.G. Alviggi ${ }^{102 a, 102 b}$, K. Amako ${ }^{66}$, P. Amaral ${ }^{29}$, C. Amelung ${ }^{22}$, V.V. Ammosov ${ }^{128}$, A. Amorim ${ }^{124 a, b}$, G. Amorós ${ }^{167}$, N. Amram ${ }^{153}$, C. Anastopoulos ${ }^{29}$, L.S. Ancu ${ }^{16}$, N. Andari ${ }^{115}$, T. Andeen $^{34}$, C.F. Anders $^{20}$, G. Anders ${ }^{58 a}$, K.J. Anderson ${ }^{30}$, A. Andreazza ${ }^{89 a, 89 b}$, V. Andrei ${ }^{58 a}$, M-L. Andrieux ${ }^{55}$, X.S. Anduaga $^{70}$, A. Angerami ${ }^{34}$, F. Anghinolfi ${ }^{29}$, A. Anisenkov ${ }^{107}$, N. Anjos ${ }^{124 a}$, A. Annovi ${ }^{47}$, A. Antonaki ${ }^{8}$, M. Antonelli $^{47}$, A. Antonov ${ }^{96}$, J. Antos ${ }^{144 b}$, F. Anulli ${ }^{132 a}$, S. Aoun ${ }^{83}$, L. Aperio Bella ${ }^{4}$, R. Apolle ${ }^{118, c}$, G. Arabidze ${ }^{88}$, I. Aracena ${ }^{143}$, Y. Arai ${ }^{66}$, A.T.H. Arce ${ }^{44}$, J.P. Archambault ${ }^{28}$, S. Arfaoui ${ }^{83}$, J-F. Arguin ${ }^{14}$, E. Arik ${ }^{18 a, *}$, M. Arik ${ }^{18 a}$, A.J. Armbruster ${ }^{87}$, O. Arnaez ${ }^{81}$, C. Arnault ${ }^{115}$, A. Artamonov ${ }^{95}$, G. Artoni ${ }^{132 a, 132 b}$, D. Arutinov ${ }^{20}$, S. Asai ${ }^{155}$, R. Asfandiyarov ${ }^{172}$, S. Ask $^{27}$, B. Åsman ${ }^{146 a, 146 b}$, L. Asquith ${ }^{5}$, K. Assamagan ${ }^{24}$, A. Astbury ${ }^{169}$, A. Astvatsatourov ${ }^{52}$, B. Aubert ${ }^{4}$, E. Auge ${ }^{115}$, K. Aug$\operatorname{sten}^{127}$, M. Aurousseau ${ }^{145 a}$, G. Avolio ${ }^{163}$, R. Avramidou ${ }^{9}$, D. Axen ${ }^{168}$, C. Ay ${ }^{54}$, G. Azuelos ${ }^{93, d}$, Y. Azuma ${ }^{155}$, M.A. Baak ${ }^{29}$, G. Baccaglioni ${ }^{89 a}$, C. Bacci ${ }^{134 a, 134 b}$, A.M. Bach ${ }^{14}$, H. Bachacou ${ }^{136}$, K. Bachas ${ }^{29}$, G. Bachy ${ }^{29}$, M. Backes ${ }^{49}$, M. Backhaus ${ }^{20}$, E. Badescu ${ }^{25 a}$, P. Bagnaia ${ }^{132 a, 132 b}$, S. Bahinipati ${ }^{2}$, Y. Bai ${ }^{32 a}$, D.C. Bailey ${ }^{158}$, T. Bain ${ }^{158}$, J.T. Baines ${ }^{129}$, O.K. Baker ${ }^{175}$, M.D. Baker ${ }^{24}$, S. Baker ${ }^{77}$, E. Banas ${ }^{38}$, P. Banerjee ${ }^{93}$, Sw. Banerjee ${ }^{172}$, D. Banfi ${ }^{29}$, A. Bangert ${ }^{150}$, V. Bansal ${ }^{169}$, H.S. Bansil $^{17}$, L. Barak ${ }^{171}$, S.P. Baranov ${ }^{94}$, A. Barashkou ${ }^{65}$, A. Barbaro Galtieri ${ }^{14}$, T. Barber ${ }^{48}$, E.L. Barberio ${ }^{86}$, D. Barberis ${ }^{50 a, 50 b}$, M. Barbero ${ }^{20}$, D.Y. Bardin ${ }^{65}$, T. Barillari ${ }^{99}$, M. Barisonzi ${ }^{174}$, T. Barklow ${ }^{143}$, N. Barlow ${ }^{27}$, B.M. Barnett ${ }^{129}$, R.M. Barnett $^{14}$, A. Baroncelli ${ }^{134 a}$, G. Barone ${ }^{49}$, A.J. Barr ${ }^{118}$, F. Barreiro ${ }^{80}$, J. Barreiro Guimarães da Costa ${ }^{57}$, P. Barrillon ${ }^{115}$, R. Bartoldus ${ }^{143}$, A.E. Barton ${ }^{71}$, V. Bartsch ${ }^{149}$, R.L. Bates ${ }^{53}$, L. Batkova ${ }^{144 a}$, J.R. Batley ${ }^{27}$, A. Battaglia ${ }^{16}$, M. Battistin $^{29}$, G. Battistoni ${ }^{89 a}$, F. Bauer ${ }^{136}$, H.S. Bawa ${ }^{143, e}$, S. Beale ${ }^{98}$, B. Beare ${ }^{158}$, T. Beau ${ }^{78}$, P.H. Beauchemin ${ }^{161}$, R. Beccherle $^{50 a}$, P. Bechtle ${ }^{20}$, H.P. Beck ${ }^{16}$, S. Becker ${ }^{98}$, M. Beckingham ${ }^{138}$, K.H. Becks ${ }^{174}$, A.J. Beddall ${ }^{18 c}$, A. Beddall ${ }^{18 c}$, S. Bedikian $^{175}$, V.A. Bednyakov ${ }^{65}$, C.P. Bee ${ }^{83}$, M. Begel ${ }^{24}$, S. Behar Harpaz ${ }^{152}$, P.K. Behera ${ }^{63}$, M. Beimforde ${ }^{99}$, C. BelangerChampagne $^{85}$, P.J. Bell ${ }^{49}$, W.H. Bell ${ }^{49}$, G. Bella ${ }^{153}$, L. Bellagamba ${ }^{19 a}$, F. Bellina ${ }^{29}$, M. Bellomo ${ }^{29}$, A. Belloni ${ }^{57}$, O. Be- $^{8}$ loborodova $^{107, \mathrm{f}}$, K. Belotskiy ${ }^{96}$, O. Beltramello ${ }^{29}$, S. Ben Ami ${ }^{152}$, O. Benary ${ }^{153}$, D. Benchekroun ${ }^{135 a}$, C. Benchouk ${ }^{83}$, M. Bendel ${ }^{81}$, N. Benekos ${ }^{165}$, Y. Benhammou ${ }^{153}$, J.A. Benitez Garcia ${ }^{159 b}$, D.P. Benjaminn ${ }^{44}$, M. Benoit ${ }^{115}$, J.R. Bensinger ${ }^{22}$, K. Benslama ${ }^{130}$, S. Bentvelsen ${ }^{105}$, D. Berge ${ }^{29}$, E. Bergeaas Kuutmann ${ }^{41}$, N. Berger ${ }^{4}$, F. Berghaus ${ }^{169}$, E. Berglund ${ }^{105}$, J. Beringer ${ }^{14}$, P. Bernat ${ }^{77}$, R. Bernhard ${ }^{48}$, C. Bernius ${ }^{24}$, T. Berry ${ }^{76}$, C. Bertella ${ }^{83}$, A. Bertin ${ }^{19 a}, 19 b$, F. Bertinelli ${ }^{29}$, F. Bertolucci ${ }^{122 a, 122 b}$, M.I. Besana ${ }^{89 a, 89 b}$, N. Besson ${ }^{136}$, S. Bethke ${ }^{99}$, W. Bhimji ${ }^{45}$, R.M. Bianchi ${ }^{29}$, M. Bianco ${ }^{72 a, 72 b}$, O. Biebel ${ }^{98}$, S.P. Bieniek ${ }^{77}$, K. Bierwagen ${ }^{54}$, J. Biesiada ${ }^{14}$, M. Biglietti ${ }^{134 a}$, H. Bilokon ${ }^{47}$, M. Bindi ${ }^{19 a, 19 b}$, S. Binet ${ }^{115}$, A. Bingul ${ }^{18 c}$, C. Bini ${ }^{132 a, 132 b}$, C. Biscarat ${ }^{177}$, U. Bitenc ${ }^{48}$, K.M. Black ${ }^{21}$, R.E. Blair ${ }^{5}$, J.-B. Blanchard ${ }^{115}$, G. Blanchot $^{29}$, T. Blazek ${ }^{144 a}$, C. Blocker ${ }^{22}$, J. Blocki ${ }^{38}$, A. Blondel ${ }^{49}$, W. Blum ${ }^{81}$, U. Blumenschein ${ }^{54}$, G.J. Bobbink ${ }^{105}$, V.B. Bobrovnikov $^{107}$, S.S. Bocchetta ${ }^{79}$, A. Bocci ${ }^{44}$, C.R. Boddy ${ }^{118}$, M. Boehler ${ }^{41}$, J. Boek ${ }^{174}$, N. Boelaert ${ }^{35}$, S. Böser ${ }^{77}$, J.A. Bogaerts $^{29}$, A. Bogdanchikov ${ }^{107}$, A. Bogouch ${ }^{90,}$, C. Bohm ${ }^{146 a}$, V. Boisvert ${ }^{76}$, T. Bold ${ }^{37}$, V. Boldea ${ }^{25 a}$, N.M. Bolnet ${ }^{136}$, M. Bona ${ }^{75}$, V.G. Bondarenko ${ }^{96}$, M. Bondioli ${ }^{163}$, M. Boonekamp ${ }^{136}$, G. Boorman ${ }^{76}$, C.N. Booth ${ }^{139}$, S. Bordoni ${ }^{78}$, C. Borer ${ }^{16}$, A. Borisov ${ }^{128}$, G. Borissov ${ }^{71}$, I. Borjanovic ${ }^{12 a}$, S. Borroni $^{87}$, K. Bos ${ }^{105}$, D. Boscherini ${ }^{19 a}$, M. Bosman ${ }^{11}$, H. Boterenbrood ${ }^{105}$, D. Botterill ${ }^{129}$, J. Bouchami ${ }^{93}$, J. Boudreau ${ }^{123}$, E.V. Bouhova-Thacker ${ }^{71}$, C. Bourdarios ${ }^{115}$, N. Bousson ${ }^{83}$, A. Boveia ${ }^{30}$, J. Boyd $^{29}$, I.R. Boyko ${ }^{65}$, N.I. Bozhko ${ }^{128}$, I. Bozovic-Jelisavcic ${ }^{12 b}$, J. Bracinik ${ }^{17}$, A. Braem ${ }^{29}$, P. Branchini ${ }^{134 a}$, G.W. Brandenburg $^{57}$, A. Brandt ${ }^{7}$, G. Brandt ${ }^{118}$, O. Brandt ${ }^{54}$, U. Bratzler ${ }^{156}$, B. Brau ${ }^{84}$, J.E. Brau ${ }^{114}$, H.M. Braun ${ }^{174}$, B. Brelier ${ }^{158}$, J. Bremer ${ }^{29}$, R. Brenner ${ }^{166}$, S. Bressler ${ }^{171}$, D. Breton ${ }^{115}$, D. Britton ${ }^{53}$, F.M. Brochu ${ }^{27}$, I. Brock ${ }^{20}$, R. Brock ${ }^{88}$, T.J. Brodbeck $^{71}$, E. Brodet ${ }^{153}$, F. Broggi ${ }^{89 a}$, C. Bromberg ${ }^{88}$, J. Bronner ${ }^{99}$, G. Brooijmans ${ }^{34}$, W.K. Brooks ${ }^{31 b}$, G. Brown ${ }^{82}$, H. Brown ${ }^{7}$, P.A. Bruckman de Renstrom ${ }^{38}$, D. Bruncko ${ }^{144 b}$, R. Bruneliere ${ }^{48}$, S. Brunet ${ }^{61}$, A. Bruni ${ }^{19 a}$, G. Bruni ${ }^{19 a}$, M. Bruschi ${ }^{19 a}$, T. Buanes ${ }^{13}$, Q. Buat ${ }^{55}$, F. Bucci ${ }^{49}$, J. Buchanan ${ }^{118}$, N.J. Buchanan ${ }^{2}$, P. Buchholz ${ }^{141}$, R.M. Buckingham ${ }^{118}$, A.G. Buckley ${ }^{45}$, S.I. Buda ${ }^{25 a}$, I.A. Budagov ${ }^{65}$, B. Budick ${ }^{108}$, V. Büscher ${ }^{81}$, L. Bugge ${ }^{117}$, D. Buira-Clark ${ }^{118}$, O. Bulekov ${ }^{96}$, M. Bunse ${ }^{42}$, T. Buran ${ }^{117}$, H. Burckhart ${ }^{29}$, S. Burdin ${ }^{73}$, T. Burgess ${ }^{13}$, S. Burke ${ }^{129}$, E. Busato ${ }^{33}$, P. Bussey ${ }^{53}$, C.P. Buszello ${ }^{166}$, F. Butin ${ }^{29}$, B. Butler ${ }^{143}$, J.M. Butler ${ }^{21}$, C.M. Buttar ${ }^{53}$, J.M. Butterworth ${ }^{77}$, W. Buttinger ${ }^{27}$, S. Cabrera Urbán ${ }^{167}$, D. Caforio ${ }^{19 a, 19 b}$, O. Cakir $^{3 a}$, P. Calafiura ${ }^{14}$, G. Calderini ${ }^{78}$, P. Calfayan ${ }^{98}$, R. Calkins ${ }^{106}$, L.P. Caloba ${ }^{23 a}$, R. Caloi ${ }^{132 a, 132 b}$, D. Calvet ${ }^{33}$, 
S. Calvet ${ }^{33}$, R. Camacho Toro ${ }^{33}$, P. Camarri ${ }^{133 a, 133 b}$, M. Cambiaghi ${ }^{119 a, 119 b}$, D. Cameron ${ }^{117}$, L.M. Caminada ${ }^{14}$, S. Campana $^{29}$, M. Campanelli ${ }^{77}$, V. Canale ${ }^{102 a, 102 b}$, F. Canelli ${ }^{30, g}$, A. Canepa ${ }^{159 a}$, J. Cantero ${ }^{80}$, L. Capasso ${ }^{102 a, 102 b}$, M.D.M. Capeans Garrido ${ }^{29}$, I. Caprini2 ${ }^{25 a}$, M. Caprinii ${ }^{25 a}$, D. Capriotti ${ }^{99}$, M. Capua ${ }^{36 a, 36 b}$, R. Caputo ${ }^{81}$, C. Caramarcu ${ }^{24}$, R. Cardarelli $^{133 a}$, T. Carlii ${ }^{29}$, G. Carlino ${ }^{102 a}$, L. Carminati ${ }^{89 a, 89 b}$, B. Caron ${ }^{85}$, S. Caron ${ }^{48}$, G.D. Carrillo Montoya ${ }^{172}$, A.A. Carter ${ }^{75}$, J.R. Carter ${ }^{27}$, J. Carvalho ${ }^{124 a, h}$, D. Casadei ${ }^{108}$, M.P. Casado ${ }^{11}$, M. Cascella ${ }^{122 a, 122 b}$, C. Caso ${ }^{50 a, 50 b, *}$, A.M. Castaneda Hernandez ${ }^{172}$, E. Castaneda-Miranda ${ }^{172}$, V. Castillo Gimenez ${ }^{167}$, N.F. Castro ${ }^{124 a}$, G. Cataldi ${ }^{72 a}$, F. Cataneo ${ }^{29}$, A. Catinaccio ${ }^{29}$, J.R. Catmore ${ }^{29}$, A. Cattai ${ }^{29}$, G. Cattani ${ }^{133 a, 133 b}$, S. Caughron ${ }^{88}$, D. Cauz ${ }^{164 a, 164 c}$, P. Cavalleri ${ }^{78}$, D. Cavalli ${ }^{89 a}$, M. CavalliSforza $^{11}$, V. Cavasinni ${ }^{122 a, 122 b}$, F. Ceradini ${ }^{134 a, 134 b}$, A.S. Cerqueira ${ }^{23 b}$, A. Cerri ${ }^{29}$, L. Cerrito ${ }^{75}$, F. Cerutti ${ }^{47}$, S.A. Cetin ${ }^{18 b}$, F. Cevenini ${ }^{102 a, 102 b}$, A. Chafaq ${ }^{135 a}$, D. Chakraborty ${ }^{106}$, K. Chan ${ }^{2}$, B. Chapleau ${ }^{85}$, J.D. Chapman ${ }^{27}$, J.W. Chapman ${ }^{87}$, ${\text { E. } \text { Chareyre }^{78} \text {, D.G. Charlton }}^{17}$, V. Chavda ${ }^{82}$, C.A. Chavez Barajas ${ }^{29}$, S. Cheatham ${ }^{85}$, S. Chekanov ${ }^{5}$, S.V. Chekulaev ${ }^{159 a}$, G.A. Chelkov ${ }^{65}$, M.A. Chelstowska ${ }^{104}$, C. $\mathrm{Chen}^{64}$, H. Chen ${ }^{24}$, S. Chen ${ }^{32 \mathrm{c}}$, T. Chen ${ }^{32 \mathrm{c}}$, X. Chen ${ }^{172}$, S. Cheng ${ }^{32 a}$, A. Cheplakov $^{65}$, V.F. Chepurnov ${ }^{65}$, R. Cherkaoui El Moursli ${ }^{135 e}$, V. Chernyatinn ${ }^{24}$, E. Cheu ${ }^{6}$, S.L. Cheung ${ }^{158}$, L. Chevalier ${ }^{136}$, G. Chiefari ${ }^{102 a, 102 b}$, L. Chikovani ${ }^{51 a}$, J.T. Childers ${ }^{58 a}$, A. Chilingarov ${ }^{71}$, G. Chiodini ${ }^{72 a}$, M.V. Chizhov ${ }^{65}$, G. Choudalakis ${ }^{30}$, S. Chouridou ${ }^{137}$, I.A. Christidi ${ }^{77}$, A. Christov ${ }^{48}$, D. Chromek-Burckhart ${ }^{29}$, M.L. Chu ${ }^{151}$, J. Chudoba ${ }^{125}$, G. Ciapetti ${ }^{132 a, 132 b}$, K. Ciba ${ }^{37}$, A.K. Ciftci ${ }^{3 a}$, R. Ciftci ${ }^{3 a}$, D. Cinca ${ }^{33}$, V. Cindro ${ }^{74}$, M.D. Ciobotaru ${ }^{163}$, C. Ciocca ${ }^{19 a}$, A. Ciocio ${ }^{14}$, M. Cirilli ${ }^{87}$, M. Citterio ${ }^{89 a}$, M. Ciubancan ${ }^{25 a}$, A. Clark ${ }^{49}$, P.J. Clark ${ }^{45}$, W. Cleland ${ }^{123}$, J.C. Clemens ${ }^{83}$, B. Clement ${ }^{55}$, C. Clement ${ }^{146 a, 146 b}$, R.W. Clifft ${ }^{129}$, Y. Coadou ${ }^{83}$, M. Cobal ${ }^{164 a, 164 c}$, A. Coccaro ${ }^{50 a, 50 b}$, J. Cochran ${ }^{64}$, P. Coe ${ }^{118}$, J.G. Cogan ${ }^{143}$, J. Coggeshall $^{165}$, E. Cogneras ${ }^{177}$, C.D. Cojocaru ${ }^{28}$, J. Colas ${ }^{4}$, A.P. Colijn ${ }^{105}$, N.J. Collins ${ }^{17}$, C. Collins-Tooth ${ }^{53}$, J. Collot ${ }^{55}$, G. Colon $^{84}$, P. Conde Muiño ${ }^{124 a}$, E. Coniavitis ${ }^{118}$, M.C. Conidi ${ }^{11}$, M. Consonni ${ }^{104}$, V. Consorti ${ }^{48}$, S. Constantinescu ${ }^{25 a}$, C. Conta ${ }^{119 a, 119 b}$, F. Conventi ${ }^{102 a, i}$, J. Cook ${ }^{29}$, M. Cooke ${ }^{14}$, B.D. Cooper ${ }^{77}$, A.M. Cooper-Sarkar ${ }^{118}$, K. Copic ${ }^{14}$, T. Cornelis$\operatorname{sen}^{174}$, M. Corradi ${ }^{19 a}$, F. Corriveau ${ }^{85, j}$, A. Cortes-Gonzalez ${ }^{165}$, G. Cortiana ${ }^{99}$, G. Costa ${ }^{89 a}$, M.J. Costa ${ }^{167}$, D. Costanzo ${ }^{139}$, T. Costin $^{30}$, D. Côté ${ }^{29}$, R. Coura Torres ${ }^{23 a}$, L. Courneyea ${ }^{169}$, G. Cowan $^{76}$, C. Cowden ${ }^{27}$, B.E. Cox ${ }^{82}$, K. Cranmer ${ }^{108}$, F. Crescioli ${ }^{122 a, 122 b}$, M. Cristinziani ${ }^{20}$, G. Crosetti ${ }^{36 a, 36 b}$, R. Crupi ${ }^{72 a, 72 b}$, S. Crépé-Renaudin ${ }^{55}$, C.-M. Cuciuc ${ }^{25 a}$, C. Cuenca Almenar ${ }^{175}$, T. Cuhadar Donszelmann ${ }^{139}$, M. Curatolo ${ }^{47}$, C.J. Curtis ${ }^{17}$, C. Cuthbert ${ }^{150}$, P. Cwetanski ${ }^{61}$, H. Czirr ${ }^{141}$, Z. Czyczula $^{175}$, S. D’Auria ${ }^{53}$, M. D’Onofrio ${ }^{73}$, A. D’Orazio ${ }^{132 a, 132 b}$, P.V.M. Da Silva ${ }^{23 a}$, C. Da Via ${ }^{82}$, W. Dabrowski ${ }^{37}$, T. Dai ${ }^{87}$, C. Dallapiccola ${ }^{84}$, M. Dam ${ }^{35}$, M. Dameri ${ }^{50 a, 50 b}$, D.S. Damiani ${ }^{137}$, H.O. Danielsson ${ }^{29}$, D. Dannheim ${ }^{99}$, V. Dao ${ }^{49}$, G. Darbo ${ }^{50 a}$, G.L. Darlea ${ }^{25 b}$, C. Daum ${ }^{105}$, W. Davey ${ }^{20}$, T. Davidek ${ }^{126}$, N. Davidson ${ }^{86}$, R. Davidson ${ }^{71}$, E. Davies ${ }^{118, c}$, M. Davies ${ }^{93}$, A.R. Davison ${ }^{77}$, Y. Davygora ${ }^{58 a}$, E. Dawe ${ }^{142}$, I. Dawson ${ }^{139}$, J.W. Dawson ${ }^{5,}$, R.K. Daya-Ishmukhametova ${ }^{39}$, K. De ${ }^{7}$, R. de Asmundis ${ }^{102 a}$, S. De Castro ${ }^{19 a, 19 b}$, P.E. De Castro Faria Salgado ${ }^{24}$, S. De Cecco $^{78}$, J. de Graat ${ }^{98}$, N. De Groot ${ }^{104}$, P. de Jong ${ }^{105}$, C. De La Taille ${ }^{115}$, H. De la Torre ${ }^{80}$, B. De Lotto ${ }^{164 a, 164 c}$, L. de Mora ${ }^{71}$, L. De Nooij ${ }^{105}$, D. De Pedis ${ }^{132 a}$, A. De Salvo ${ }^{132 a}$, U. De Sanctis ${ }^{164 a, 164 c}$, A. De Santo ${ }^{149}$, J.B. De Vivie De Regie ${ }^{115}$, S. Dean ${ }^{77}$, W.J. Dearnaley $^{71}$, R. Debbe ${ }^{24}$, C. Debenedetti ${ }^{45}$, D.V. Dedovich ${ }^{65}$, J. Degenhardt ${ }^{120}$, M. Dehchar ${ }^{118}$, C. Del Papa ${ }^{164 a, 164 c}$, J. Del Peso ${ }^{80}$, T. Del Prete ${ }^{122 a, 122 b}$, T. Delemontex ${ }^{55}$, M. Deliyergiyev ${ }^{74}$, A. Dell'Acqua ${ }^{29}$, L. Dell'Asta ${ }^{21}$, M. Della Pietra ${ }^{102 a, i}$, D. della Volpe ${ }^{102 a, 102 b}$, M. Delmastro ${ }^{4}$, N. Delruelle ${ }^{29}$, P.A. Delsart ${ }^{55}$, C. Deluca ${ }^{148}$, S. Demers ${ }^{175}$, M. Demichev ${ }^{65}$, B. Demirkoz ${ }^{11, \mathrm{k}}$, J. Deng ${ }^{163}$, S.P. Denisov ${ }^{128}$, D. Derendarz ${ }^{38}$, J.E. Derkaoui ${ }^{135 d}$, F. Derue ${ }^{78}$, P. Dervan ${ }^{73}$, K. De$\mathrm{sch}^{20}$, E. Devetak ${ }^{148}$, P.O. Deviveiros ${ }^{158}$, A. Dewhurst ${ }^{129}$, B. DeWilde ${ }^{148}$, S. Dhaliwal ${ }^{158}$, R. Dhullipudi ${ }^{24,1}$, A. Di Ciaccio $^{133 a, 133 b}$, L. Di Ciaccio ${ }^{4}$, A. Di Girolamo ${ }^{29}$, B. Di Girolamo ${ }^{29}$, S. Di Luise ${ }^{134 a, 134 b}$, A. Di Mattia ${ }^{172}$, B. Di Micco ${ }^{29}$, R. Di Nardo ${ }^{47}$, A. Di Simone ${ }^{133 a, 133 b}$, R. Di Sipio ${ }^{19 a, 19 b}$, M.A. Diaz ${ }^{31 a}$, F. Diblen ${ }^{18 c}$, E.B. Diehl ${ }^{87}$, J. Dietrich ${ }^{41}$, T.A. Diet-

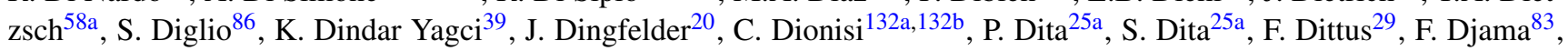
T. Djobava ${ }^{51 b}$, M.A.B. do Vale ${ }^{23 c}$, A. Do Valle Wemans ${ }^{124 a}$, T.K.O. Doan ${ }^{4}$, M. Dobbs ${ }^{85}$, R. Dobinson ${ }^{29, *}$, D. Dobos ${ }^{29}$, E. Dobson ${ }^{29, m}$, J. Dodd ${ }^{34}$, C. Doglioni ${ }^{118}$, T. Doherty ${ }^{53}$, Y. Doi ${ }^{66, *}$, J. Dolejsi ${ }^{126}$, I. Dolenc ${ }^{74}$, Z. Dolezal ${ }^{126}$, B.A. Dolgoshein $^{96, *}$, T. Dohmae ${ }^{155}$, M. Donadelli2 ${ }^{23 d}$, M. Donega ${ }^{120}$, J. Donini ${ }^{33}$, J. Dopke ${ }^{29}$, A. Doria ${ }^{102 a}$, A. Dos Anjos ${ }^{172}$, M. Dosil ${ }^{11}$, A. Dotti ${ }^{122 a, 122 b}$, M.T. Dova ${ }^{70}$, J.D. Dowell ${ }^{17}$, A.D. Doxiadis ${ }^{105}$, A.T. Doyle ${ }^{53}$, Z. Drasal ${ }^{126}$, J. Drees ${ }^{174}$, N. Dressnandt ${ }^{120}$, H. Drevermann ${ }^{29}$, C. Driouichi ${ }^{35}$, M. Dris ${ }^{9}$, J. Dubbert ${ }^{99}$, S. Dube ${ }^{14}$, E. Duchovni ${ }^{171}$, G. Duckeck ${ }^{98}$, A. Dudarev ${ }^{29}$, F. Dudziak ${ }^{64}$, M. Dührssen ${ }^{29}$, I.P. Duerdoth ${ }^{82}$, L. Duflot ${ }^{115}$, M-A. Dufour ${ }^{85}$, M. Dunford ${ }^{29}$, H. Duran Yildiz $^{3 a}$, R. Duxfield ${ }^{139}$, M. Dwuznik ${ }^{37}$, F. Dydak ${ }^{29}$, M. Düren ${ }^{52}$, W.L. Ebenstein ${ }^{44}$, J. Ebke ${ }^{98}$, S. Eckweiler ${ }^{81}$, K. Edmonds $^{81}$, C.A. Edwards ${ }^{76}$, N.C. Edwards ${ }^{53}$, W. Ehrenfeld ${ }^{41}$, T. Ehrich ${ }^{99}$, T. Eifert $^{29}$, G. Eigen ${ }^{13}$, K. Einsweiler ${ }^{14}$, E. Eisenhandler $^{75}$, T. Ekelof ${ }^{166}$, M. El Kacimi ${ }^{135 c}$, M. Ellert ${ }^{166}$, S. Elles ${ }^{4}$, F. Ellinghaus ${ }^{81}$, K. Ellis ${ }^{75}$, N. Ellis ${ }^{29}$, J. Elmsheuser ${ }^{98}$, M. Elsing ${ }^{29}$, D. Emeliyanov ${ }^{129}$, R. Engelmann ${ }^{148}$, A. Engl ${ }^{98}$, B. Epp ${ }^{62}$, A. Eppig ${ }^{87}$, J. Erdmann ${ }^{54}$, A. Ereditato ${ }^{16}$, D. Eriksson $^{146 a}$, J. Ernst ${ }^{1}$, M. Ernst ${ }^{24}$, J. Ernwein ${ }^{136}$, D. Errede ${ }^{165}$, S. Errede ${ }^{165}$, E. Ertel ${ }^{81}$, M. Escalier ${ }^{115}$, C. Escobar ${ }^{123}$, X. Espinal Curull ${ }^{11}$, B. Esposito ${ }^{47}$, F. Etienne ${ }^{83}$, A.I. Etienvre ${ }^{136}$, E. Etzion ${ }^{153}$, D. Evangelakou ${ }^{54}$, H. Evans ${ }^{61}$, L. Fabbri ${ }^{19 a, 19 b}$, C. Fabre $^{29}$, R.M. Fakhrutdinov ${ }^{128}$, S. Falciano ${ }^{132 a}$, Y. Fang ${ }^{172}$, M. Fanti ${ }^{89,89 b}$, A. Farbin ${ }^{7}$, A. Farilla ${ }^{134 a}$, J. Farley ${ }^{148}$, T. Farooque $^{158}$, S.M. Farrington ${ }^{118}$, P. Farthouat ${ }^{29}$, P. Fassnacht ${ }^{29}$, D. Fassouliotis ${ }^{8}$, B. Fatholahzadeh ${ }^{158}$, A. Favareto ${ }^{89 a, 89 b}$, 
L. Fayard ${ }^{115}$, S. Fazio ${ }^{36 a, 36 b}$, R. Febbraro ${ }^{33}$, P. Federic ${ }^{144 a}$, O.L. Fedin ${ }^{121}$, W. Fedorko ${ }^{88}$, M. Fehling-Kaschek ${ }^{48}$, L. Feligioni $^{83}$, D. Fellmann ${ }^{5}$, C. Feng ${ }^{32 d}$, E.J. Feng ${ }^{30}$, A.B. Fenyuk ${ }^{128}$, J. Ferencei ${ }^{144 b}$, J. Ferland ${ }^{93}$, W. Fernando ${ }^{109}$, S. Ferrag $^{53}$, J. Ferrando ${ }^{53}$, V. Ferrara ${ }^{41}$, A. Ferrari ${ }^{166}$, P. Ferrari ${ }^{105}$, R. Ferrari ${ }^{19 a}$, A. Ferrer ${ }^{167}$, M.L. Ferrer ${ }^{47}$, D. Ferrere ${ }^{49}$, C. Ferretti ${ }^{87}$, A. Ferretto Parodi ${ }^{50 a, 50 b}$, M. Fiascaris ${ }^{30}$, F. Fiedler ${ }^{81}$, A. Filipčic ${ }^{74}$, A. Filippas 9 , F. Filthaut ${ }^{104}$, M. FinckeKeeler $^{169}$, M.C.N. Fiolhais ${ }^{124 a, h}$, L. Fiorini ${ }^{167}$, A. Firan ${ }^{39}$, G. Fischer ${ }^{41}$, P. Fischer $^{20}$, M.J. Fisher ${ }^{109}$, M. Flechl ${ }^{48}$, I. Fleck ${ }^{141}$, J. Fleckner ${ }^{81}$, P. Fleischmann ${ }^{173}$, S. Fleischmann ${ }^{174}$, T. Flick ${ }^{174}$, L.R. Flores Castillo ${ }^{172}$, M.J. Flowerdew ${ }^{99}$, M. Fokitis ${ }^{9}$, T. Fonseca Martin ${ }^{16}$, J. Fopma ${ }^{118}$, D.A. Forbush ${ }^{138}$, A. Formica ${ }^{166}$, A. Forti ${ }^{82}$, D. Fortin ${ }^{159 a}$, J.M. Foster ${ }^{82}$, D. Fournier ${ }^{115}$, A. Foussat ${ }^{29}$, A.J. Fowler ${ }^{44}$, K. Fowler ${ }^{137}$, H. Fox ${ }^{71}$, P. Francavilla ${ }^{122 a, 122 b}$, S. Franchino ${ }^{119 a, 119 b}$, D. Francis ${ }^{29}$, T. Frank ${ }^{171}$, M. Franklin ${ }^{57}$, S. Franz ${ }^{29}$, M. Fraternali ${ }^{119 a, 119 b}$, S. Fratina ${ }^{120}$, S.T. French ${ }^{27}$, F. Friedrich ${ }^{43}$, R. Froeschl ${ }^{29}$, D. Froidevaux $^{29}$, J.A. Frost $^{27}$, C. Fukunaga ${ }^{156}$, E. Fullana Torregrosa ${ }^{29}$, J. Fuster ${ }^{167}$, C. Gabaldon ${ }^{29}$, O. Gabizon ${ }^{171}$, T. Gadfort $^{24}$, S. Gadomski ${ }^{49}$, G. Gagliardi ${ }^{50,50 b}$, P. Gagnon ${ }^{61}$, C. Galea ${ }^{98}$, E.J. Gallas ${ }^{118}$, V. Gallo ${ }^{16}$, B.J. Gallop ${ }^{129}$, P. Gallus $^{125}$, K.K. Gan $^{109}$, Y.S. Gao ${ }^{143, e}$, V.A. Gapienko ${ }^{128}$, A. Gaponenko ${ }^{14}$, F. Garberson ${ }^{175}$, M. Garcia-Sciveres ${ }^{14}$, C. García $^{167}$, J.E. García Navarro ${ }^{167}$, R.W. Gardner ${ }^{30}$, N. Garelli ${ }^{29}$, H. Garitaonandia ${ }^{105}$, V. Garonne ${ }^{29}$, J. Garvey ${ }^{17}$, C. Gatti ${ }^{47}$, G. Gaudio ${ }^{119 a}$, O. Gaumer ${ }^{49}$, B. Gaur ${ }^{141}$, L. Gauthier ${ }^{136}$, I.L. Gavrilenko ${ }^{94}$, C. Gay ${ }^{168}$, G. Gaycken ${ }^{20}$, J-C. Gayde ${ }^{29}$, E.N. Gazis ${ }^{9}$, P. Ge ${ }^{32 d}$, C.N.P. Gee ${ }^{129}$, D.A.A. Geerts ${ }^{105}$, Ch. Geich-Gimbel ${ }^{20}$, K. Gellerstedt ${ }^{146 a, 146 b}$, C. Gemme ${ }^{50 a}$, A. Gemmell $^{53}$, M.H. Genest ${ }^{98}$, S. Gentile ${ }^{132 a, 132 b}$, M. George ${ }^{54}$, S. George ${ }^{76}$, P. Gerlach $^{174}$, A. Gershon ${ }^{153}$, C. Geweniger ${ }^{58 a}$, H. Ghazlane ${ }^{135 b}$, N. Ghodbane $e^{33}$, B. Giacobbe ${ }^{19 a}$, S. Giagu ${ }^{132 a, 132 b}$, V. Giakoumopoulou ${ }^{8}$, V. Giangiobbe ${ }^{11}$, F. Gianotti $^{29}$, B. Gibbard ${ }^{24}$, A. Gibson ${ }^{158}$, S.M. Gibson ${ }^{29}$, L.M. Gilbert ${ }^{118}$, V. Gilewsky ${ }^{91}$, D. Gillberg ${ }^{28}$, A.R. Gillman ${ }^{129}$, D.M. Gingrich ${ }^{2, d}$, J. Ginzburg ${ }^{153}$, N. Giokaris ${ }^{8}$, M.P. Giordani ${ }^{164 c}$, R. Giordano ${ }^{102 a, 102 b}$, F.M. Giorgi ${ }^{15}$, P. Giovannini ${ }^{99}$, P.F. Giraud ${ }^{136}$, D. Giugni ${ }^{89 a}$, M. Giunta ${ }^{93}$, P. Giusti ${ }^{19 a}$, B.K. Gjelsten ${ }^{117}$, L.K. Gladilin ${ }^{97}$, C. Glasman ${ }^{80}$, J. Glatzer ${ }^{48}$, A. Glazov ${ }^{41}$, K.W. Glitza ${ }^{174}$, G.L. Glonti ${ }^{65}$, J. Godfrey ${ }^{142}$, J. Godlewski ${ }^{29}$, M. Goebel ${ }^{41}$, T. Göpfert ${ }^{43}$, C. Goeringer ${ }^{81}$, C. Gössling ${ }^{42}$, T. Göttfert ${ }^{99}$, S. Goldfarb ${ }^{87}$, T. Golling ${ }^{175}$, S.N. Golovnia ${ }^{128}$, A. Gomes ${ }^{124 a, b}$, L.S. Gomez Fajardo ${ }^{41}$, R. Gonçalo ${ }^{76}$, J. Goncalves Pinto Firmino Da Costa ${ }^{41}$, L. Gonella ${ }^{20}$, A. Gonidec ${ }^{29}$, S. Gonzalez ${ }^{172}$, S. González de la Hoz ${ }^{167}$, G. Gonzalez Parra ${ }^{11}$, M.L. Gonzalez Silva ${ }^{26}$, S. Gonzalez-Sevilla ${ }^{49}$, J.J. Goodson ${ }^{148}$, L. Goossens ${ }^{29}$, P.A. Gorbounov ${ }^{95}$, H.A. Gordon ${ }^{24}$, I. Gorelov ${ }^{103}$, G. Gorfine ${ }^{174}$, B. Gorini ${ }^{29}$, E. Gorini ${ }^{72 a, 72 b}$, A. Gorišek ${ }^{74}$, E. Gornicki ${ }^{38}$, S.A. Gorokhov ${ }^{128}$, V.N. Goryachev ${ }^{128}$, B. Gosdzik ${ }^{41}$, M. Gosselink ${ }^{105}$, M.I. Gostkin ${ }^{65}$, I. Gough Eschrich ${ }^{163}$, M. Gouighri ${ }^{135 a}$, D. Goujdami $^{135 c}$, M.P. Goulette ${ }^{49}$, A.G. Goussiou ${ }^{138}$, C. Goy ${ }^{4}$, S. Gozpinar ${ }^{22}$, I. Grabowska-Bold ${ }^{37}$, P. Grafström ${ }^{29}$, K-J. Grahn ${ }^{41}$, F. Grancagnolo ${ }^{72 a}$, S. Grancagnolo ${ }^{15}$, V. Grassi ${ }^{148}$, V. Gratchev ${ }^{121}$, N. Grau ${ }^{34}$, H.M. Gray ${ }^{29}$, J.A. Gray ${ }^{148}$, E. Graziani ${ }^{134 a}$, O.G. Grebenyuk ${ }^{121}$, T. Greenshaw ${ }^{73}$, Z.D. Greenwood ${ }^{24,1}$, K. Gregersen ${ }^{35}$, I.M. Gregor ${ }^{41}$, P. Grenier ${ }^{143}$, J. Griffiths ${ }^{138}$, N. Grigalashvili ${ }^{65}$, A.A. Grillo ${ }^{137}$, S. Grinstein ${ }^{11}$, Y.V. Grishkevich ${ }^{97}$, J.-F. Grivaz ${ }^{115}$, M. Groh ${ }^{99}$, E. Gross ${ }^{171}$, J. GrosseKnetter $^{54}$, J. Groth-Jensen ${ }^{171}$, K. Grybel ${ }^{141}$, V.J. Guarino ${ }^{5}$, D. Guest ${ }^{175}$, C. Guicheney ${ }^{33}$, A. Guida ${ }^{72 a, 72 b}$, S. Guindon ${ }^{54}$, H. Guler ${ }^{85, n}$, J. Gunther ${ }^{125}$, B. Guo ${ }^{158}$, J. Guo ${ }^{34}$, A. Gupta ${ }^{30}$, Y. Gusakov ${ }^{65}$, V.N. Gushchin ${ }^{128}$, A. Gutierrez ${ }^{93}$, P. Gutierrez $^{111}$, N. Guttman ${ }^{153}$, O. Gutzwiller ${ }^{172}$, C. Guyot ${ }^{136}$, C. Gwenlan ${ }^{118}$, C.B. Gwilliam ${ }^{73}$, A. Haas ${ }^{143}$, S. Haas ${ }^{29}$, C. Haber ${ }^{14}$,

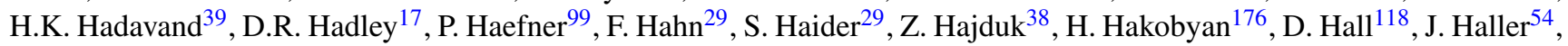
K. Hamacher ${ }^{174}$, P. Hamal ${ }^{113}$, M. Hamer ${ }^{54}$, A. Hamilton ${ }^{145 b, o}$, S. Hamilton ${ }^{161}$, H. Han ${ }^{32 a}$, L. Han ${ }^{32 b}$, K. Hanagaki ${ }^{116}$,

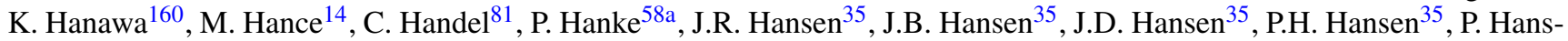

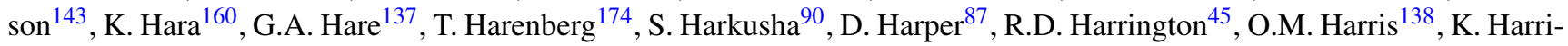
son $^{17}$, J. Hartert ${ }^{48}$, F. Hartjes ${ }^{105}$, T. Haruyama ${ }^{66}$, A. Harvey ${ }^{56}$, S. Hasegawa ${ }^{101}$, Y. Hasegawa ${ }^{140}$, S. Hassani ${ }^{136}$, M. Hatch ${ }^{29}$, D. Hauff ${ }^{99}$, S. Haug ${ }^{16}$, M. Hauschild ${ }^{29}$, R. Hauser ${ }^{88}$, M. Havranek ${ }^{20}$, B.M. Hawes ${ }^{118}$, C.M. Hawkes ${ }^{17}$, R.J. Hawkings $^{29}$, D. Hawkins ${ }^{163}$, T. Hayakawa ${ }^{67}$, T. Hayashi ${ }^{160}$, D. Hayden ${ }^{76}$, H.S. Hayward ${ }^{73}$, S.J. Haywood ${ }^{129}$, E. Hazen ${ }^{21}$, M. He ${ }^{32 d}$, S.J. Head ${ }^{17}$, V. Hedberg ${ }^{79}$, L. Heelan ${ }^{7}$, S. Heim ${ }^{88}$, B. Heinemann ${ }^{14}$, S. Heisterkamp ${ }^{35}$, L. Helary ${ }^{4}$, C. Heller ${ }^{98}$, M. Heller ${ }^{29}$, S. Hellman ${ }^{146 a, 146 b}$, D. Hellmich ${ }^{20}$, C. Helsens ${ }^{11}$, R.C.W. Henderson ${ }^{71}$, M. Henke ${ }^{58 a}$, A. Henrichs ${ }^{54}$, A.M. Henriques Correia ${ }^{29}$, S. Henrot-Versille ${ }^{115}$, F. Henry-Couannier ${ }^{83}$, C. Hensel ${ }^{54}$, T. Hen $\beta^{174}$, C.M. Hernandez ${ }^{7}$, Y. Hernández Jiménez $^{167}$, R. Herrberg ${ }^{15}$, A.D. Hershenhorn ${ }^{152}$, G. Herten ${ }^{48}$, R. Hertenberger ${ }^{98}$, L. Hervas ${ }^{29}$, N.P. Hessey ${ }^{105}$, E. HigónRodriguez $^{167}$, D. Hill ${ }^{5, *}$, J.C. Hill ${ }^{27}$, N. Hill ${ }^{5}$, K.H. Hiller ${ }^{41}$, S. Hillert ${ }^{20}$, S.J. Hillier ${ }^{17}$, I. Hinchliffe ${ }^{14}$, E. Hines ${ }^{120}$, M. Hi$\operatorname{rose}^{116}$, F. Hirsch ${ }^{42}$, D. Hirschbueh1 ${ }^{174}$, J. Hobbs ${ }^{148}$, N. Hod ${ }^{153}$, M.C. Hodgkinson ${ }^{139}$, P. Hodgson ${ }^{139}$, A. Hoecker ${ }^{29}$, M.R. Hoeferkamp ${ }^{103}$, J. Hoffman ${ }^{39}$, D. Hoffmann ${ }^{83}$, M. Hohlfeld ${ }^{81}$, M. Holder ${ }^{141}$, S.O. Holmgren ${ }^{146 a}$, T. Holy ${ }^{127}$, J.L. Holzbauer ${ }^{88}$, Y. Homma ${ }^{67}$, T.M. Hong ${ }^{120}$, L. Hooft van Huysduynen ${ }^{108}$, T. Horazdovsky ${ }^{127}$, C. Horn ${ }^{143}$, S. Horner ${ }^{48}$, J-Y. Hostachy ${ }^{55}$, S. Hou ${ }^{151}$, M.A. Houlden ${ }^{73}$, A. Hoummada ${ }^{135 a}$, J. Howarth ${ }^{82}$, D.F. Howell ${ }^{118}$, I. Hristova ${ }^{15}$, J. Hrivnac ${ }^{115}$, I. Hruska ${ }^{125}$, T. Hryn'ova ${ }^{4}$, P.J. Hsu ${ }^{81}$, S.-C. Hsu ${ }^{14}$, G.S. Huang ${ }^{111}$, Z. Hubacek ${ }^{127}$, F. Hubaut ${ }^{83}$, F. Huegging ${ }^{20}$, T.B. Huff$\operatorname{man}^{118}$, E.W. Hughes ${ }^{34}$, G. Hughes ${ }^{71}$, R.E. Hughes-Jones ${ }^{82}$, M. Huhtinen ${ }^{29}$, P. Hurst ${ }^{57}$, M. Hurwitz ${ }^{14}$, U. Husemann ${ }^{41}$, N. Huseynov ${ }^{65, p}$, J. Huston ${ }^{88}$, J. Huth ${ }^{57}$, G. Iacobucci ${ }^{49}$, G. Iakovidis ${ }^{9}$, M. Ibbotson ${ }^{82}$, I. Ibragimov ${ }^{141}$, R. Ichimiya ${ }^{67}$, L. Iconomidou-Fayard ${ }^{115}$, J. Idarraga ${ }^{115}$, P. Iengo ${ }^{102 a}$, O. Igonkina ${ }^{105}$, Y. Ikegami ${ }^{66}$, M. Ikeno ${ }^{66}$, Y. Ilchenko ${ }^{39}$, D. Iliadis $^{154}$, N. Ilic ${ }^{158}$, D. Imbault ${ }^{78}$, M. Imori ${ }^{155}$, T. Ince ${ }^{20}$, J. Inigo-Golfin ${ }^{29}$, P. Ioannou ${ }^{8}$, M. Iodice ${ }^{134 a}$, A. Irles Quiles ${ }^{167}$, 
C. Isaksson ${ }^{166}$, A. Ishikawa ${ }^{67}$, M. Ishino ${ }^{68}$, R. Ishmukhametov ${ }^{39}$, C. Issever ${ }^{118}$, S. Istin ${ }^{18 a}$, A.V. Ivashin ${ }^{128}$, W. Iwanski ${ }^{38}$, H. Iwasaki ${ }^{66}$, J.M. Izen ${ }^{40}$, V. Izzo ${ }^{102 a}$, B. Jackson ${ }^{120}$, J.N. Jackson ${ }^{73}$, P. Jackson ${ }^{143}$, M.R. Jaekel ${ }^{29}$, V. Jain ${ }^{61}$, K. Jakobs ${ }^{48}$, S. Jakobsen ${ }^{35}$, J. Jakubek ${ }^{127}$, D.K. Jana ${ }^{111}$, E. Jankowski ${ }^{158}$, E. Jansen ${ }^{77}$, H. Jansen ${ }^{29}$, A. Jantsch ${ }^{99}$, M. Janus ${ }^{20}$, G. Jarl$\operatorname{skog}^{79}$, L. Jeanty ${ }^{57}$, K. Jelen ${ }^{37}$, I. Jen-La Plante ${ }^{30}$, P. Jenni ${ }^{29}$, A. Jeremie ${ }^{4}$, P. Jež ${ }^{35}$, S. Jézéquel ${ }^{4}$, M.K. Jha ${ }^{19 a}$, H. Ji $^{172}$,

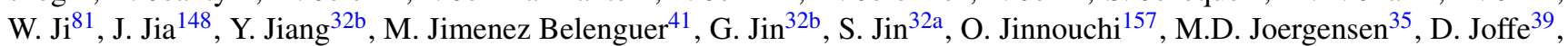
L.G. Johansen ${ }^{13}$, M. Johansen ${ }^{146 a, 146 b}$, K.E. Johansson ${ }^{146 a}$, P. Johansson ${ }^{139}$, S. Johnert ${ }^{41}$, K.A. Johns ${ }^{6}$, K. Jon-And ${ }^{146 a, 146 b}$, G. Jones $^{82}$, R.W.L. Jones ${ }^{71}$, T.W. Jones ${ }^{77}$, T.J. Jones ${ }^{73}$, O. Jonsson ${ }^{29}$, C. Joram ${ }^{29}$, P.M. Jorge ${ }^{124 a}$, J. Joseph ${ }^{14}$, T. Jovin ${ }^{12 b}$, X. Ju ${ }^{172}$, C.A. Jung ${ }^{42}$, V. Juranek ${ }^{125}$, P. Jussel ${ }^{62}$, A. Juste Rozas ${ }^{11}$, V.V. Kabachenko ${ }^{128}$, S. Kabana ${ }^{16}$, M. Kaci ${ }^{167}$, A. Kaczmarska ${ }^{38}$, P. Kadlecik ${ }^{35}$, M. Kado ${ }^{115}$, H. Kagan ${ }^{109}$, M. Kagan ${ }^{57}$, S. Kaiser ${ }^{99}$, E. Kajomovitz ${ }^{152}$, S. Kalinin ${ }^{174}$, L.V. Kalinovskaya ${ }^{65}$, S. Kama ${ }^{39}$, N. Kanaya ${ }^{155}$, M. Kaneda ${ }^{29}$, T. Kanno ${ }^{157}$, V.A. Kantserov ${ }^{96}$, J. Kanzaki ${ }^{66}$, B. Kaplan ${ }^{175}$, A. Kapliy ${ }^{30}$, J. Kaplon ${ }^{29}$, D. $\mathrm{Kar}^{43}$, M. Karagounis ${ }^{20}$, M. Karagoz ${ }^{18}$, M. Karnevskiy ${ }^{41}$, K. Karr ${ }^{5}$, V. Kartvelishvili ${ }^{71}$, A.N. Karyukhin ${ }^{128}$, L. Kashif ${ }^{172}$, G. Kasieczka ${ }^{58 b}$, R.D. Kass ${ }^{109}$, A. Kastanas ${ }^{13}$, M. Kataoka ${ }^{4}$, Y. Kataoka ${ }^{155}$, E. Katsoufis ${ }^{9}$, J. Katzy ${ }^{41}$, V. Kaushik ${ }^{6}$, K. Kawagoe ${ }^{67}$, T. Kawamoto ${ }^{155}$, G. Kawamura ${ }^{81}$, M.S. Kayl ${ }^{105}$, V.A. Kazanin ${ }^{107}$, M.Y. Kazarinov $^{65}$, J.R. Keates ${ }^{82}$, R. Keeler ${ }^{169}$, R. Kehoe ${ }^{39}$, M. Keil ${ }^{54}$, G.D. Kekelidze ${ }^{65}$, J. Kennedy ${ }^{98}$, C.J. Kenney ${ }^{143}$, M. Kenyon ${ }^{53}$, O. Kepka ${ }^{125}$, N. Kerschen ${ }^{29}$, B.P. Kerševan ${ }^{74}$, S. Kersten ${ }^{174}$, K. Kessoku ${ }^{155}$, J. Keung ${ }^{158}$, F. Khalil-zada ${ }^{10}$, H. Khandanyan $^{165}$, A. Khanov ${ }^{112}$, D. Kharchenko ${ }^{65}$, A. Khodinov ${ }^{96}$, A.G. Kholodenko ${ }^{128}$, A. Khomich ${ }^{58 a}$, T.J. Khoo ${ }^{27}$, G. Khoriauli $^{20}$, A. Khoroshilov ${ }^{174}$, N. Khovanskiy ${ }^{65}$, V. Khovanskiy ${ }^{95}$, E. Khramov ${ }^{65}$, J. Khubua ${ }^{51 b}$, H. Kim ${ }^{146 a, 146 b}$, M.S. Kim ${ }^{2}$, P.C. $\mathrm{Kim}^{143}$, S.H. Kim ${ }^{160}$, N. Kimura ${ }^{170}$, O. Kind ${ }^{15}$, B.T. King ${ }^{73}$, M. King ${ }^{67}$, R.S.B. King ${ }^{118}$, J. Kirk ${ }^{129}$, L.E. Kirsch ${ }^{22}$, A.E. Kiryunin ${ }^{99}$, T. Kishimoto ${ }^{67}$, D. Kisielewska ${ }^{37}$, T. Kittelmann ${ }^{123}$, A.M. Kiver ${ }^{128}$, E. Kladiva ${ }^{144 b}$, J. Klaiber-Lodewigs ${ }^{42}$, M. Klein ${ }^{73}$, U. Klein ${ }^{73}$, K. Kleinknecht ${ }^{81}$, M. Klemetti ${ }^{85}$, A. Klier ${ }^{171}$, A. Klimentov ${ }^{24}$, R. Klingenberg ${ }^{42}$, E.B. Klinkby ${ }^{35}$, T. Klioutchnikova ${ }^{29}$, P.F. Klok ${ }^{104}$, S. Klous ${ }^{105}$, E.-E. Kluge ${ }^{58 a}$, T. Kluge ${ }^{73}$, P. Kluit ${ }^{105}$, S. Kluth ${ }^{99}$, N.S. Knecht ${ }^{158}$, E. Kneringer $^{62}$, J. Knobloch ${ }^{29}$, E.B.F.G. Knoops ${ }^{83}$, A. Knue ${ }^{54}$, B.R. Ko ${ }^{44}$, T. Kobayashi ${ }^{155}$, M. Kobel ${ }^{43}$, M. Kocian ${ }^{143}$, P. Kodys ${ }^{126}$, K. Köneke ${ }^{29}$, A.C. König ${ }^{104}$, S. Koenig ${ }^{81}$, L. Köpke ${ }^{81}$, F. Koetsveld ${ }^{104}$, P. Koevesarki ${ }^{20}$, T. Koffas ${ }^{28}$, E. Koffeman ${ }^{105}$, F. $K{ }^{54}{ }^{54}$, Z. Kohout ${ }^{127}$, T. Kohriki ${ }^{66}$, T. Koi ${ }^{143}$, T. Kokott ${ }^{20}$, G.M. Kolachev ${ }^{107}$, H. Kolanoski ${ }^{15}$, V. Kolesnikov ${ }^{65}$, I. Koletsou $^{89 a}$, J. Koll ${ }^{88}$, D. Kollar ${ }^{29}$, M. Kollefrath ${ }^{48}$, S.D. Kolya ${ }^{82}$, A.A. Komar ${ }^{94}$, Y. Komori ${ }^{155}$, T. Kondo ${ }^{66}$, T. Kono ${ }^{41, q}$, A.I. Kononov ${ }^{48}$, R. Konoplich ${ }^{108, r}$, N. Konstantinidis 77 , A. Kootz ${ }^{174}$, S. Koperny ${ }^{37}$, S.V. Kopikov ${ }^{128}$, K. Korcyl ${ }^{38}$, K. Kordas ${ }^{154}$, V. Koreshev ${ }^{128}$, A. Korn ${ }^{118}$, A. Korol ${ }^{107}$, I. Korolkov ${ }^{11}$, E.V. Korolkova ${ }^{139}$, V.A. Korotkov ${ }^{128}$, O. Kortner $^{99}$, S. Kortner ${ }^{99}$, V.V. Kostyukhin ${ }^{20}$, M.J. Kotamäki ${ }^{29}$, S. Kotov ${ }^{99}$, V.M. Kotov ${ }^{65}$, A. Kotwal ${ }^{44}$, C. Kourkoumelis ${ }^{8}$, V. Kouskoura ${ }^{154}$, A. Koutsman ${ }^{159}$, R. Kowalewski ${ }^{169}$, T.Z. Kowalski ${ }^{37}$, W. Kozanecki ${ }^{136}$, A.S. Kozhin ${ }^{128}$, V. Kral ${ }^{127}$, V.A. Kramarenko ${ }^{97}$, G. Kramberger ${ }^{74}$, M.W. Krasny ${ }^{78}$, A. Krasznahorkay ${ }^{108}$, J. Kraus ${ }^{88}$, J.K. Kraus ${ }^{20}$, A. Kreisel ${ }^{153}$, F. Krejci ${ }^{127}$, J. Kretzschmar ${ }^{73}$, N. Krieger ${ }^{54}$, P. Krieger ${ }^{158}$, K. Kroeninger ${ }^{54}$, H. Kroha ${ }^{99}$, J. Kroll ${ }^{120}$, J. Kroseberg ${ }^{20}$, J. Krstic ${ }^{12 a}$, U. Kruchonak ${ }^{65}$, H. Krüger ${ }^{20}$, T. Kruker ${ }^{16}$, N. Krumnack ${ }^{64}$, Z.V. Krumshteyn ${ }^{65}$, A. Kruth ${ }^{20}$, T. Kubota ${ }^{86}$, S. Kuehn ${ }^{48}$, A. Kugel ${ }^{58 c}$, T. Kuhl ${ }^{41}$, D. Kuhn ${ }^{62}$, V. Kukhtin ${ }^{65}$, Y. Kulchitsky ${ }^{90}$, S. Kuleshov ${ }^{31 b}$, C. Kummer ${ }^{98}$, M. Kuna ${ }^{78}$, N. Kundu ${ }^{118}$, J. Kunkle ${ }^{120}$, A. Kupco ${ }^{125}$, H. Kurashige ${ }^{67}$, M. Kurata ${ }^{160}$, Y.A. Kurochkin ${ }^{90}$, V. Kus ${ }^{125}$, M. Kuze ${ }^{157}$, J. Kvita ${ }^{142}$, R. Kwee ${ }^{15}$, A. La Rosa ${ }^{49}$, L. La Rotonda ${ }^{36 a, 36 b}$, L. Labarga ${ }^{80}$, J. Labbe ${ }^{4}$, S. Lablak ${ }^{135 a}$, C. Lacasta ${ }^{167}$, F. La-

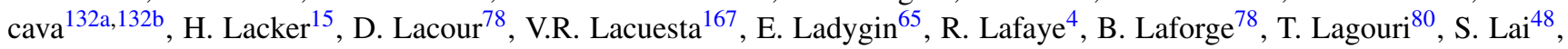
E. Laisne ${ }^{55}$, M. Lamanna ${ }^{29}$, C.L. Lampen ${ }^{6}$, W. Lampl $^{6}$, E. Lancon ${ }^{136}$, U. Landgraf ${ }^{48}$, M.P.J. Landon ${ }^{75}$, H. Landsman ${ }^{152}$, J.L. Lane ${ }^{82}$, C. Lange ${ }^{41}$, A.J. Lankford ${ }^{163}$, F. Lanni ${ }^{24}$, K. Lantzsch ${ }^{174}$, S. Laplace ${ }^{78}$, C. Lapoire $^{20}$, J.F. Laporte ${ }^{136}$, T. Lari ${ }^{89 a}$, A.V. Larionov ${ }^{128}$, A. Larner ${ }^{118}$, C. Lasseur ${ }^{29}$, M. Lassnig ${ }^{29}$, P. Laurelli ${ }^{47}$, W. Lavrijsen ${ }^{14}$, P. Laycock ${ }^{73}$, A.B. Lazarev ${ }^{65}$, O. Le Dortz ${ }^{78}$, E. Le Guirriec ${ }^{83}$, C. Le Maner ${ }^{158}$, E. Le Menedeu ${ }^{136}$, C. Lebel ${ }^{93}$, T. LeCompte ${ }^{5}$, F. Ledroit-Guillon ${ }^{55}$, H. Lee ${ }^{105}$, J.S.H. Lee ${ }^{116}$, S.C. Lee ${ }^{151}$, L. Lee ${ }^{175}$, M. Lefebvre ${ }^{169}$, M. Legendre ${ }^{136}$, A. Leger ${ }^{49}$, B.C. LeGeyt ${ }^{120}$, F. Legger $^{98}$, C. Leggett ${ }^{14}$, M. Lehmacher ${ }^{20}$, G. Lehmann Miotto ${ }^{29}$, X. Lei ${ }^{6}$, M.A.L. Leite ${ }^{23 d}$, R. Leitner ${ }^{126}$, D. Lellouch ${ }^{171}$, M. Leltchouk ${ }^{34}$, B. Lemmer ${ }^{54}$, V. Lendermann ${ }^{58 a}$, K.J.C. Leney ${ }^{145 b}$, T. Lenz ${ }^{105}$, G. Lenzen ${ }^{174}$, B. Lenzi ${ }^{29}$, K. Leonhardt ${ }^{43}$, S. Leontsinis ${ }^{9}$, C. Leroy ${ }^{93}$, J-R. Lessard ${ }^{169}$, J. Lesser ${ }^{146 a}$, C.G. Lester ${ }^{27}$, A. Leung Fook Cheong ${ }^{172}$, J. Levêque ${ }^{4}$, D. Levin ${ }^{87}$, L.J. Levinson ${ }^{171}$, M.S. Levitski ${ }^{128}$, A. Lewis ${ }^{118}$, G.H. Lewis ${ }^{108}$, A.M. Leyko ${ }^{20}$, M. Leyton ${ }^{15}$, B. Li ${ }^{83}, \mathrm{H}^{2} \mathrm{Li}^{172, \mathrm{~s}}, \mathrm{~S}^{\mathrm{L}} \mathrm{Li}^{32 \mathrm{~b}, \mathrm{t}}$,

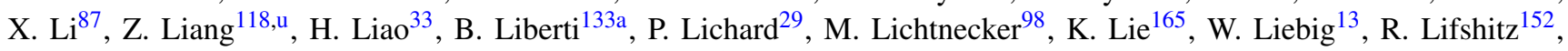
C. Limbach $^{20}$, A. Limosani ${ }^{86}$, M. Limper ${ }^{63}$, S.C. Lin $^{151, v}$, F. Linde ${ }^{105}$, J.T. Linnemann ${ }^{88}$, E. Lipeles ${ }^{120}$, L. Lipinsky ${ }^{125}$,

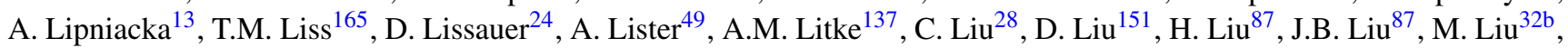
S. Liu ${ }^{2}$, Y. Liu ${ }^{32 b}$, M. Livan119a,119b, S.S.A. Livermore ${ }^{118}$, A. Lleres ${ }^{55}$, J. Llorente Merino ${ }^{80}$, S.L. Lloyd ${ }^{75}$, E. Lobodzinska ${ }^{41}$, P. Loch ${ }^{6}$, W.S. Lockman ${ }^{137}$, T. Loddenkoetter ${ }^{20}$, F.K. Loebinger ${ }^{82}$, A. Loginov ${ }^{175}$, C.W. Loh ${ }^{168}$, T. Lohse ${ }^{15}$, K. Lohwasser ${ }^{48}$, M. Lokajicek ${ }^{125}$, J. Loken ${ }^{118}$, V.P. Lombardo ${ }^{4}$, R.E. Long ${ }^{71}$, L. Lopes ${ }^{124 a, b}$, D. Lopez Mateos ${ }^{57}$, J. Lorenz ${ }^{98}$, M. Losada ${ }^{162}$, P. Loscutoff ${ }^{14}$, F. Lo Sterzo ${ }^{132 a, 132 b}$, M.J. Losty ${ }^{159 a}$, X. Lou $^{40}$, A. Lounis ${ }^{115}$, K.F. Loureiro ${ }^{162}$, J. Love ${ }^{21}$, P.A. Love ${ }^{71}$, A.J. Lowe ${ }^{143, e}$, F. Lu ${ }^{32 a}$, H.J. Lubatti ${ }^{138}$, C. Luci ${ }^{132 a, 132 b}$, A. Lucotte ${ }^{55}$, A. Ludwig ${ }^{43}$, D. Ludwig ${ }^{41}$, I. Ludwig ${ }^{48}$, J. Lud- 
wig $^{48}$, F. Luehring ${ }^{61}$, G. Luijckx ${ }^{105}$, D. Lumb ${ }^{48}$, L. Luminari ${ }^{132 a}$, E. Lund ${ }^{117}$, B. Lund-Jensen ${ }^{147}$, B. Lundberg ${ }^{79}$, J. Lundberg ${ }^{146 a, 146 b}$, J. Lundquist ${ }^{35}$, M. Lungwitz ${ }^{81}$, G. Lutz ${ }^{99}$, D. Lynn ${ }^{24}$, J. Lys ${ }^{14}$, E. Lytken ${ }^{79}$, H. Ma ${ }^{24}$, L.L. Ma ${ }^{172}$, J.A. Macana Goia $^{93}$, G. Maccarrone ${ }^{47}$, A. Macchiolo ${ }^{99}$, B. Maček ${ }^{74}$, J. Machado Miguens ${ }^{124 a}$, R. Mackeprang ${ }^{35}$, R.J. Madaras ${ }^{14}$, W.F. Mader ${ }^{43}$, R. Maenner ${ }^{58 c}$, T. Maeno ${ }^{24}$, P. Mättig ${ }^{174}$, S. Mättig ${ }^{41}$, L. Magnoni ${ }^{29}$, E. Magradze ${ }^{54}$, Y. Mahalalel ${ }^{153}$, K. Mahboubi $^{48}$, G. Mahout ${ }^{17}$, C. Maiani ${ }^{132 a, 132 b}$, C. Maidantchik ${ }^{23 a}$, A. Maio ${ }^{124 a, b}$, S. Majewskii ${ }^{24}$, Y. Makida ${ }^{66}$, N. Makovec ${ }^{115}$, P. $\mathrm{Mal}^{136}$, Pa. Malecki ${ }^{38}$, P. Malecki ${ }^{38}$, V.P. Maleev ${ }^{121}$, F. Malek ${ }^{55}$, U. Mallik ${ }^{63}$, D. Malon ${ }^{5}$, C. Malone ${ }^{143}$, S. Maltezos $^{9}$, V. Malyshev ${ }^{107}$, S. Malyukov ${ }^{29}$, R. Mameghani ${ }^{98}$, J. Mamuzic ${ }^{12 b}$, A. Manabe ${ }^{66}$, L. Mandellii ${ }^{89}$, I. Mandić ${ }^{74}$, R. Mandrysch ${ }^{15}$, J. Maneira ${ }^{124 a}$, P.S. Mangeard ${ }^{88}$, I.D. Manjavidze ${ }^{65}$, A. Mann ${ }^{54}$, P.M. Manning ${ }^{137}$, A. ManousakisKatsikakis $^{8}$, B. Mansoulie ${ }^{136}$, A. Manz ${ }^{99}$, A. Mapelli ${ }^{29}$, L. Mapelli ${ }^{29}$, L. March ${ }^{80}$, J.F. Marchand ${ }^{28}$, F. Marchese ${ }^{133 a, 133 b}$, G. Marchiori ${ }^{78}$, M. Marcisovsky ${ }^{125}$, A. Marin ${ }^{21, *}$, C.P. Marino ${ }^{169}$, F. Marroquim²3a, R. Marshall ${ }^{82}$, Z. Marshall ${ }^{29}$, F.K. Martens ${ }^{158}$, S. Marti-Garcia ${ }^{167}$, A.J. Martin ${ }^{175}$, B. $\operatorname{Martin}^{29}$, B. Martin ${ }^{88}$, F.F. Martin ${ }^{120}$, J.P. Martin ${ }^{93}$, Ph. Mar$\operatorname{tin}^{55}$, T.A. Martin ${ }^{17}$, V.J. Martin ${ }^{45}$, B. Martin dit Latour ${ }^{49}$, S. Martin-Haugh ${ }^{149}$, M. Martinez ${ }^{11}$, V. Martinez Outschoorn ${ }^{57}$, A.C. Martyniuk ${ }^{169}$, M. Marx ${ }^{82}$, F. Marzano ${ }^{132 a}$, A. Marzin ${ }^{111}$, L. Masetti ${ }^{81}$, T. Mashimo ${ }^{155}$, R. Mashinistov ${ }^{94}$, J. Masik $^{82}$, A.L. Maslennikov ${ }^{107}$, I. Massa ${ }^{19 a, 19 b}$, G. Massaro ${ }^{105}$, N. Massol $^{4}$, P. Mastrandrea ${ }^{132 a, 132 b}$, A. Mastroberardino ${ }^{36 a, 36 b}$, T. Masubuchi $^{155}$, M. Mathes ${ }^{20}$, P. Matricon ${ }^{115}$, H. Matsumoto ${ }^{155}$, H. Matsunaga ${ }^{155}$, T. Matsushita ${ }^{67}$, C. Mattravers ${ }^{118, c}$, J.M. Maugain $^{29}$, J. Maurer ${ }^{83}$, S.J. Maxfield ${ }^{73}$, D.A. Maximov ${ }^{107, f}$, E.N. May ${ }^{5}$, A. Mayne ${ }^{139}$, R. Mazini ${ }^{151}$, M. Mazur ${ }^{20}$, M. Mazzanti $^{89 a}$, E. Mazzoni ${ }^{122 a, 122 b}$, S.P. Mc Kee ${ }^{87}$, A. McCarn ${ }^{165}$, R.L. McCarthy ${ }^{148}$, T.G. McCarthy ${ }^{28}$, N.A. McCubbin ${ }^{129}$, K.W. McFarlane ${ }^{56}$, J.A. Mcfayden ${ }^{139}$, H. McGlone ${ }^{53}$, G. Mchedlidze ${ }^{51 b}$, R.A. McLaren ${ }^{29}$, T. Mclaughlan ${ }^{17}$, S.J. McMahon $^{129}$, R.A. McPherson ${ }^{169, j}$, A. Meade ${ }^{84}$, J. Mechnich ${ }^{105}$, M. Mechtel ${ }^{174}$, M. Medinnis ${ }^{41}$, R. Meera-Lebbai ${ }^{111}$, T. Meguro $^{116}$, R. Mehdiyev ${ }^{93}$, S. Mehlhase ${ }^{35}$, A. Mehta ${ }^{73}$, K. Meier ${ }^{58 a}$, B. Meirose ${ }^{79}$, C. Melachrinos ${ }^{30}$, B.R. Mellado Garcia $^{172}$, L. Mendoza Navas ${ }^{162}$, Z. Meng ${ }^{151, \mathrm{~s}}$, A. Mengarelli ${ }^{19 a, 19 b}$, S. Menke ${ }^{99}$, C. Menot $^{29}$, E. Meoni ${ }^{11}$, K.M. Mercurio ${ }^{57}$, P. Mermod $^{49}$, L. Merola ${ }^{102 a, 102 b}$, C. Meroni ${ }^{89 a}$, F.S. Merritt ${ }^{30}$, A. Messina ${ }^{29}$, J. Metcalfe ${ }^{103}$, A.S. Mete ${ }^{64}$, C. Meyer ${ }^{81}$, C. $M_{e y e r}{ }^{30}$, J-P. Meyer ${ }^{136}$, J. Meyer ${ }^{173}$, J. Meyer ${ }^{54}$, T.C. Meyer $^{29}$, W.T. Meyer ${ }^{64}$, J. Miao ${ }^{32 d}$, S. Michal ${ }^{29}$, L. Micu ${ }^{25 a}$, R.P. Middleton ${ }^{129}$, P. Miele ${ }^{29}$, S. Migas ${ }^{73}$, L. Mijović ${ }^{41}$, G. Mikenberg ${ }^{171}$, M. Mikestikova ${ }^{125}$, M. Mikuž ${ }^{74}$, D.W. Miller ${ }^{30}$, R.J. Miller ${ }^{88}$, W.J. Mills ${ }^{168}$, C. Mills ${ }^{57}$, A. Milov ${ }^{171}$, D.A. Milstead ${ }^{146 a, 146 b}$, D. Milstein ${ }^{171}$, A.A. Minaenko ${ }^{128}$, M. Miñano Moya $^{167}$, I.A. Minashvili ${ }^{65}$, A.I. Mincer ${ }^{108}$, B. Mindur $^{37}$, M. Mineev ${ }^{65}$, Y. Ming ${ }^{172}$, L.M. Mir ${ }^{11}$, G. Mirabelli ${ }^{132 a}$, L. Miralles Verge ${ }^{11}$, A. Misiejuk ${ }^{76}$, J. Mitrevski ${ }^{137}$, G.Y. Mitrofanov ${ }^{128}$, V.A. Mitsou ${ }^{167}$, S. Mitsui ${ }^{66}$, P.S. Miyagawa ${ }^{139}$, K. Miyazaki ${ }^{67}$, J.U. Mjörnmark ${ }^{79}$, T. Moa ${ }^{146 a, 146 b}$, P. Mockett ${ }^{138}$, S. Moed ${ }^{57}$, V. Moeller ${ }^{27}$, K. Mönig ${ }^{41}$, N. Möser ${ }^{20}$, S. Mohapatra ${ }^{148}$, W. Mohr ${ }^{48}$, S. Mohrdieck-Möck ${ }^{99}$, A.M. Moisseev ${ }^{128,{ }^{*}}$, R. Moles-Valls ${ }^{167}$, J. Molina-Perez ${ }^{29}$, J. Monk ${ }^{77}$, E. Monnier ${ }^{83}$, S. Montesano ${ }^{89 a, 89 b}$, F. Monticellii ${ }^{70}$, S. Monzani ${ }^{19 a, 19 b}$, R.W. Moore ${ }^{2}$, G.F. Moorhead ${ }^{86}$, C. Mora Herrera ${ }^{49}$,

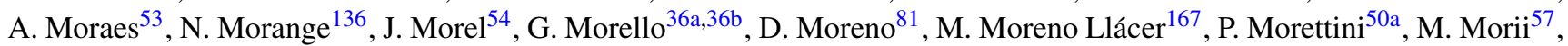
J. Morin ${ }^{75}$, A.K. Morley ${ }^{29}$, G. Mornacchi ${ }^{29}$, S.V. Morozov ${ }^{96}$, J.D. Morris ${ }^{75}$, L. Morvaj ${ }^{101}$, H.G. Moser ${ }^{99}$, M. Mosidze ${ }^{51 b}$, J. Moss $^{109}$, R. Mount ${ }^{143}$, E. Mountricha ${ }^{9, w}$, S.V. Mouraviev ${ }^{94}$, E.J.W. Moyse ${ }^{84}$, M. Mudrinic ${ }^{12 b}$, F. Mueller ${ }^{58 a}$, J. Mueller ${ }^{123}$, K. Mueller ${ }^{20}$, T.A. Müller ${ }^{9}$, T. Mueller ${ }^{81}$, D. Muenstermann ${ }^{29}$, A. Muir ${ }^{168}$, Y. Munwes ${ }^{153}$, W.J. Murray ${ }^{129}$, I. Mussche ${ }^{105}$, E. Musto ${ }^{102 a, 102 b}$, A.G. Myagkov ${ }^{128}$, M. Myska ${ }^{125}$, J. Nadal ${ }^{11}$, K. Nagai ${ }^{160}$, K. Nagano ${ }^{66}$, Y. Nagasaka ${ }^{60}$, A.M. Nairz ${ }^{29}$, Y. Nakahama ${ }^{29}$, K. Nakamura ${ }^{155}$, T. Nakamura ${ }^{155}$, I. Nakano ${ }^{110}$, G. Nanava ${ }^{20}$, A. Napier ${ }^{161}$, M. Nash ${ }^{77, \mathrm{c}}$, N.R. Nation ${ }^{21}$, T. Nattermann ${ }^{20}$, T. Naumann ${ }^{41}$, G. Navarro ${ }^{162}$, H.A. Neal ${ }^{87}$, E. Nebot ${ }^{80}$, P.Yu. Nechaeva ${ }^{94}$, A. Negri ${ }^{119 a, 119 b}$, G. Negri $^{29}$, S. Nektarijevic ${ }^{49}$, A. Nelson ${ }^{163}$, S. Nelson ${ }^{143}$, T.K. Nelson ${ }^{143}$, S. Nemecek ${ }^{125}$, P. Nemethy ${ }^{108}$, A.A. Nepomuceno ${ }^{23 a}$, M. Nessi ${ }^{29, x}$, M.S. Neubauer ${ }^{165}$, A. Neusiedl ${ }^{81}$, R.M. Neves ${ }^{108}$, P. Nevski ${ }^{24}$, P.R. Newman ${ }^{17}$, V. Nguyen Thi Hong ${ }^{136}$, R.B. Nickerson ${ }^{118}$, R. Nicolaidou ${ }^{136}$, L. Nicolas ${ }^{139}$, B. Nicquevert ${ }^{29}$, F. Niedercorn ${ }^{115}$, J. Nielsen ${ }^{137}$, T. Niinikoski ${ }^{29}$, N. Nikiforou ${ }^{34}$, A. Nikiforov ${ }^{15}$, V. Nikolaenko ${ }^{128}$, K. Nikolaev ${ }^{65}$, I. Nikolic-Audit ${ }^{78}$, K. Nikolics ${ }^{49}$, K. Nikolopoulos ${ }^{24}$, H. Nilsen ${ }^{48}$, P. Nilsson ${ }^{7}$, Y. Ninomiya ${ }^{155}$, A. Nisati ${ }^{132 a}$, T. Nishiyama ${ }^{67}$, R. Nisius ${ }^{99}$, L. Nodulman ${ }^{5}$, M. Nomachi ${ }^{116}$, I. Nomidis $^{154}$, M. Nordberg ${ }^{29}$, B. Nordkvist ${ }^{146 a, 146 b}$, P.R. Norton $^{129}$, J. Novakova ${ }^{126}$, M. Nozaki ${ }^{66}$, L. Nozka ${ }^{113}$, I.M. Nugent ${ }^{159 a}$, A.-E. Nuncio-Quiroz ${ }^{20}$, G. Nunes Hanninger ${ }^{86}$, T. Nunnemann ${ }^{98}$, E. Nurse ${ }^{77}$, T. Nyman ${ }^{29}$, B.J. O'Brien ${ }^{45}$, S.W. O'Neale ${ }^{17, *}$, D.C. O'Neil ${ }^{142}$, V. O’Shea ${ }^{53}$, F.G. Oakham ${ }^{28, d}$, H. Oberlack ${ }^{99}$, J. Ocariz ${ }^{78}$, A. Ochi' ${ }^{67}$, S. Oda ${ }^{155}$, S. Odaka ${ }^{66}$, J. Odier ${ }^{83}$, H. Ogren ${ }^{61}$, A. Oh $^{82}$, S.H. Oh ${ }^{44}$, C.C. Ohm ${ }^{146 a, 146 b}$, T. Ohshima ${ }^{101}$, H. Ohshita ${ }^{140}$, T. Ohsugi ${ }^{59}$, S. Okada ${ }^{67}$, H. Okawa ${ }^{163}$, Y. Okumura ${ }^{101}$, T. Okuyama ${ }^{155}$, A. Olariu ${ }^{25 a}$, M. Olcese ${ }^{50 a}$, A.G. Olchevski ${ }^{65}$, M. Oliveira ${ }^{124 a, h}$, D. Oliveira Damazio ${ }^{24}$, E. Oliver Garcia ${ }^{167}$, D. Olivito ${ }^{120}$, A. Olszewski ${ }^{38}$, J. Olszowska ${ }^{38}$, C. Omachi ${ }^{67}$, A. Onofre ${ }^{124 a, y}$, P.U.E. Onyisi ${ }^{30}$, C.J. Oram ${ }^{159 a}$, M.J. Oreglia ${ }^{30}$, Y. Oren ${ }^{153}$, D. Orestano ${ }^{134 a, 134 b}$, I. Orlov ${ }^{107}$, C. Oropeza Barrera ${ }^{53}$, R.S. Orr ${ }^{158}$, B. Osculati $^{50 a, 50 b}$, R. Ospanov ${ }^{120}$, C. Osuna ${ }^{11}$, G. Otero y Garzon ${ }^{26}$, J.P. Ottersbach ${ }^{105}$, M. Ouchrif ${ }^{135 d}$, F. Ould-Saada ${ }^{117}$, A. Ouraou ${ }^{136}$, Q. Ouyang ${ }^{32 a}$, M. Owen ${ }^{82}$, S. Owen ${ }^{139}$, V.E. Ozcan ${ }^{18 a}$, N. Ozturk ${ }^{7}$, A. Pacheco Pages ${ }^{11}$, C. Padilla Aranda ${ }^{11}$, S. Pagan Griso ${ }^{14}$, E. Paganis ${ }^{139}$, F. Paige ${ }^{24}$, P. Pais ${ }^{84}$, K. Pajchel ${ }^{117}$, G. Palacino ${ }^{159 b}$, C.P. Paleari ${ }^{6}$, S. Palestini ${ }^{29}$, D. Pallin ${ }^{33}$, A. Palma ${ }^{124 a}$, J.D. Palmer ${ }^{17}$, Y.B. Pan ${ }^{172}$, E. Panagiotopoulou ${ }^{9}$, B. Panes ${ }^{31 a}$, N. Panikashvilii ${ }^{87}$, S. Panitkin ${ }^{24}$, D. Pantea ${ }^{25 a}$, 
M. Panuskova ${ }^{125}$, V. Paolone ${ }^{123}$, A. Papadelis ${ }^{146 a}$, Th.D. Papadopoulou ${ }^{9}$, A. Paramonov ${ }^{5}$, W. Park ${ }^{24, z}$, M.A. Parker ${ }^{27}$, F. Parodi ${ }^{50 a, 50 b}$, J.A. Parsons ${ }^{34}$, U. Parzefall ${ }^{48}$, E. Pasqualucci ${ }^{132 a}$, A. Passeri ${ }^{134 a}$, F. Pastore ${ }^{134 a, 134 b}$, Fr. Pastore ${ }^{76}$, G. Pásztor $^{49, \text { aa }}$, S. Pataraia ${ }^{174}$, N. Patel ${ }^{150}$, J.R. Pater ${ }^{82}$, S. Patricelli ${ }^{102 a, 102 b}$, T. Pauly ${ }^{29}$, M. Pecsy ${ }^{144 a}$, M.I. Pedraza Morales ${ }^{172}$, S.V. Peleganchuk ${ }^{107}$, H. Peng ${ }^{32 b}$, R. Pengo ${ }^{29}$, A. Penson ${ }^{34}$, J. Penwell ${ }^{61}$, M. Perantoni2 ${ }^{23 a}$, K. Perez ${ }^{34, a b}$, T. Perez Cavalcanti $^{41}$, E. Perez Codina ${ }^{11}$, M.T. Pérez García-Estañ ${ }^{167}$, V. Perez Reale ${ }^{34}$, L. Perini ${ }^{89 a, 89 b}$, H. Pernegger ${ }^{29}$, R. Perrino ${ }^{72 a}$, P. Perrodo ${ }^{4}$, S. Persembe ${ }^{3 a}$, A. Perus ${ }^{115}$, V.D. Peshekhonov ${ }^{65}$, B.A. Petersen ${ }^{29}$, J. Petersen ${ }^{29}$, T.C. Petersen ${ }^{35}$, E. Petit ${ }^{4}$, A. Petridis ${ }^{154}$, C. Petridou ${ }^{154}$, E. Petrolo ${ }^{132 a}$, F. Petrucci ${ }^{134 a, 134 b}$, D. Petschull ${ }^{41}$, M. Petteni ${ }^{142}$, R. Pezoa ${ }^{31 b}$, A. Phan $^{86}$, P.W. Phillips ${ }^{129}$, G. Piacquadio ${ }^{29}$, E. Piccaro ${ }^{75}$, M. Piccinini ${ }^{19 a, 19 b}$, S.M. Piec $^{41}$, R. Piegaia ${ }^{26}$, D.T. Pignotti ${ }^{109}$, J.E. Pilcher ${ }^{30}$, A.D. Pilkington ${ }^{82}$, J. Pina ${ }^{124 a, b}$, M. Pinamonti ${ }^{164 a, 164 c}$, A. Pinder ${ }^{118}$, J.L. Pinfold ${ }^{2}$, J. Ping ${ }^{32 c}$, B. Pinto ${ }^{124 a, b}$, O. Pirotte ${ }^{29}$, C. Pizio ${ }^{89 a, 89 b}$, M. Plamondon ${ }^{169}$, M.-A. Pleier ${ }^{24}$, A.V. Pleskach ${ }^{128}$, A. Poblaguev ${ }^{24}$, S. Poddar ${ }^{58 a}$, F. Podlyski ${ }^{33}$, L. Poggioli $^{115}$, T. Poghosyan ${ }^{20}$, M. Pohl ${ }^{49}$, F. Polci ${ }^{55}$, G. Polesello ${ }^{119 a}$, A. Policicchio ${ }^{36 a, 36 b}$, A. Polini ${ }^{19 a}$, J. Poll ${ }^{75}$, V. Polychronakos $^{24}$, D.M. Pomarede ${ }^{136}$, D. Pomeroy ${ }^{22}$, K. Pommès ${ }^{29}$, L. Pontecorvo ${ }^{132 a}$, B.G. Pope ${ }^{88}$, G.A. Popeneciu ${ }^{25 a}$, D.S. Popovic ${ }^{12 a}$, A. Poppleton ${ }^{29}$, X. Portell Bueso ${ }^{29}$, C. Posch $^{21}$, G.E. Pospelov ${ }^{99}$, S. Pospisil ${ }^{127}$, I.N. Potrap ${ }^{99}$, C.J. Potter $^{149}$, C.T. Potter ${ }^{114}$, G. Poulard ${ }^{29}$, J. Poveda ${ }^{172}$, R. Prabhu ${ }^{77}$, P. Pralavorio ${ }^{83}$, A. Pranko ${ }^{14}$, S. Prasad ${ }^{57}$, R. Prava$\operatorname{han}^{7}$, S. Prell ${ }^{64}$, K. Pretzl ${ }^{16}$, L. Pribyl ${ }^{29}$, D. Price ${ }^{61}$, J. Price ${ }^{73}$, L.E. Price ${ }^{5}$, M.J. Price ${ }^{29}$, D. Prieur ${ }^{123}$, M. Primavera $^{72 a}$, K. Prokofiev ${ }^{108}$, F. Prokoshin ${ }^{31 b}$, S. Protopopescu ${ }^{24}$, J. Proudfoot ${ }^{5}$, X. Prudent ${ }^{43}$, H. Przysiezniak ${ }^{4}$, S. Psoroulas ${ }^{20}$, E. Ptacek ${ }^{114}$, E. Pueschel ${ }^{84}$, J. Purdham ${ }^{87}$, M. Purohit ${ }^{24, \text {, }}$, P. Puzo ${ }^{115}$, Y. Pylypchenko ${ }^{63}$, J. Qian ${ }^{87}$, Z. Qian ${ }^{83}$, Z. Qin ${ }^{41}$, A. Quadt ${ }^{54}$, D.R. Quarrie ${ }^{14}$, W.B. Quayle ${ }^{172}$, F. Quinonez ${ }^{31 a}$, M. Raas ${ }^{104}$, V. Radescu ${ }^{58 b}$, B. Radics ${ }^{20}$, T. Rador ${ }^{18 a}$, F. Ragusa $^{89 a, 89 b}$, G. Rahal ${ }^{177}$, A.M. Rahimi ${ }^{109}$, D. Rahm ${ }^{24}$, S. Rajagopalan ${ }^{24}$, M. Rammensee ${ }^{48}$, M. Rammes ${ }^{141}$, M. Ramstedt $^{146 a, 146 b}$, A.S. Randle-Conde ${ }^{39}$, K. Randrianarivony ${ }^{28}$, P.N. Ratoff ${ }^{71}$, F. Rauscher ${ }^{98}$, M. Raymond ${ }^{29}$, A.L. Read ${ }^{117}$, D.M. Rebuzzi ${ }^{119 a, 119 b}$, A. Redelbach ${ }^{173}$, G. Redlinger ${ }^{24}$, R. Reece ${ }^{120}$, K. Reeves ${ }^{40}$, A. Reichold ${ }^{105}$, E. Reinherz-Aronis ${ }^{153}$, A. Reinsch ${ }^{114}$, I. Reisinger ${ }^{42}$, D. Reljic ${ }^{12 a}$, C. Rembser ${ }^{29}$, Z.L. Ren ${ }^{151}$, A. Renaud ${ }^{115}$, P. Renkel ${ }^{39}$, M. Rescigno ${ }^{132 a}$, S. Resconi ${ }^{89}$, B. Resende ${ }^{136}$, P. Reznicek ${ }^{98}$, R. Rezvani ${ }^{158}$, A. Richards ${ }^{77}$, R. Richter ${ }^{99}$, E. Richter-Was ${ }^{4, a c}$, M. Ridel $^{78}$, M. Rijpstra ${ }^{105}$, M. Rijssenbeek ${ }^{148}$, A. Rimoldi ${ }^{19 a, 119 b}$, L. Rinaldi ${ }^{19 a}$, R.R. Rios ${ }^{39}$, I. Riu ${ }^{11}$, G. Rivoltella ${ }^{89 a, 89 b}$, F. Rizatdinova $^{112}$, E. Rizvi ${ }^{75}$, S.H. Robertson ${ }^{85}$, , A. Robichaud-Veronneau ${ }^{118}$, D. Robinson ${ }^{27}$, J.E.M. Robinson ${ }^{77}$, M. Robinson ${ }^{114}$, A. Robson ${ }^{53}$, J.G. Rocha de Lima ${ }^{106}$, C. Roda ${ }^{122 a, 122 b}$, D. Roda Dos Santos ${ }^{29}$, S. Rodier ${ }^{80}$, D. Rodriguez ${ }^{162}$, Y. Rodriguez Garcia $^{162}$, A. Roe ${ }^{54}$, S. Roe ${ }^{29}$, O. Røhne ${ }^{117}$, V. Rojo ${ }^{1}$, S. Rolli ${ }^{161}$, A. Romaniouk ${ }^{96}$, M. Romano ${ }^{19 a}$, 19b , V.M. Romanov ${ }^{65}$, G. $R_{0 m e}{ }^{26}$, L. Roos $^{78}$, E. Ros ${ }^{167}$, S. Rosati ${ }^{132 a}$, K. Rosbach ${ }^{49}$, A. Rose ${ }^{149}$, M. Rose ${ }^{76}$, G.A. Rosenbaum ${ }^{158}$, E.I. Rosenberg $^{64}$, P.L. Rosendahl ${ }^{13}$, O. Rosenthal ${ }^{141}$, L. Rosselet ${ }^{49}$, V. Rossetti ${ }^{11}$, E. Rossi ${ }^{132 a, 132 b}$, L.P. Rossi ${ }^{50 a}$, M. Rotaru ${ }^{25 a}$, I. Roth ${ }^{171}$, J. Rothberg ${ }^{138}$, D. Rousseau ${ }^{115}$, C.R. Royon ${ }^{136}$, A. Rozanov ${ }^{83}$, Y. Rozen ${ }^{152}$, X. Ruan ${ }^{115, \text { ad }}$, I. Rubinskiy ${ }^{41}$, B. Ruckert ${ }^{98}$, N. Ruckstuhl ${ }^{105}$, V.I. Rud ${ }^{97}$, C. Rudolph ${ }^{43}$, G. Rudolph ${ }^{62}$, F. Rühr ${ }^{6}$, F. Ruggieri ${ }^{134 a, 134 b}$, A. Ruiz-Martinez ${ }^{64}$, V. Rumiantsev ${ }^{91, *}$, L. Rumyantsev ${ }^{65}$, K. Runge ${ }^{48}$, O. Runolfsson ${ }^{20}$, Z. Rurikova ${ }^{48}$, N.A. Rusakovich ${ }^{65}$, D.R. Rust ${ }^{61}$, J.P. Rutherfoord ${ }^{6}$, C. Ruwiedel ${ }^{14}$, P. Ruzicka ${ }^{125}$, Y.F. Ryabov ${ }^{121}$, V. Ryadovikov ${ }^{128}$, P. Ryan ${ }^{88}$, M. Rybar ${ }^{126}$, G. Rybkin ${ }^{115}$, N.C. Ryder ${ }^{118}$, S. Rzaeva ${ }^{10}$, A.F. Saavedra ${ }^{150}$, I. Sadeh ${ }^{153}$, H.F-W. Sadrozinski ${ }^{137}$, R. Sadykov ${ }^{65}$, F. Safai Tehrani ${ }^{132 a}$, H. Sakamoto ${ }^{155}$, G. Salamanna ${ }^{75}$, A. Salamon ${ }^{133 a}$, M. Saleem ${ }^{111}$, D. Salihagic ${ }^{99}$, A. Salnikov ${ }^{143}$, J. Salt ${ }^{167}$, B.M. Salvachua Ferrando $^{5}$, D. Salvatore ${ }^{36 a, 36 b}$, F. Salvatore ${ }^{149}$, A. Salvucci ${ }^{104}$, A. Salzburger ${ }^{29}$, D. Sampsonidis ${ }^{154}$, B.H. Samset ${ }^{117}$, A. Sanchez ${ }^{102 a, 102 b}$, H. Sandaker ${ }^{13}$, H.G. Sander ${ }^{81}$, M.P. Sanders ${ }^{98}$, M. Sandhoff ${ }^{174}$, T. Sandoval ${ }^{27}$, C. Sandoval ${ }^{162}$, R. Sandstroem $^{99}$, S. Sandvoss ${ }^{174}$, D.P.C. Sankey ${ }^{129}$, A. Sansoni ${ }^{47}$, C. Santamarina Rios ${ }^{85}$, C. Santoni ${ }^{33}$, R. Santonico ${ }^{133 a, 133 b}$, H. Santos ${ }^{124 a}$, J.G. Saraiva ${ }^{124 a}$, T. Sarangi ${ }^{172}$, E. Sarkisyan-Grinbaum ${ }^{7}$, F. Sarri ${ }^{12 a, 122 b}$, G. Sartisohn ${ }^{174}$, O. Sasaki ${ }^{66}$, N. Sasao $^{68}$, I. Satsounkevitch ${ }^{90}$, G. Sauvage ${ }^{4}$, E. Sauvan ${ }^{4}$, J.B. Sauvan ${ }^{115}$, P. Savard ${ }^{158, d}$, V. Savinov ${ }^{123}$, D.O. Savu ${ }^{29}$, L. Sawyer ${ }^{24,1}$, D.H. Saxon ${ }^{53}$, L.P. Says ${ }^{33}$, C. Sbarra ${ }^{19 a}$, A. Sbrizzi19a,19b, O. Scallon ${ }^{93}$, D.A. Scannicchio ${ }^{163}$, M. Scarcella $^{150}$, J. Schaarschmidt ${ }^{115}$, P. Schacht ${ }^{99}$, U. Schäfer ${ }^{81}$, S. Schaepe ${ }^{20}$, S. Schaetzel ${ }^{58 b}$, A.C. Schaffer ${ }^{115}$, D. Schaile ${ }^{98}$, R.D. Schamberger ${ }^{148}$, A.G. Schamov ${ }^{107}$, V. Scharf ${ }^{58 a}$, V.A. Schegelsky ${ }^{121}$, D. Scheirich ${ }^{87}$, M. Schernau ${ }^{163}$, M.I. Scherzer ${ }^{34}$, C. Schiavi ${ }^{50 a, 50 b}$, J. Schieck ${ }^{98}$, M. Schioppa ${ }^{36 a, 36 b}$, S. Schlenker ${ }^{29}$, J.L. Schlereth ${ }^{5}$, E. Schmidt ${ }^{48}$, K. Schmieden ${ }^{20}$, C. Schmitt ${ }^{81}$, S. Schmitt ${ }^{58 b}$, M. Schmitz ${ }^{20}$, A. Schöning ${ }^{58 b}$, M. Schott ${ }^{29}$, D. Schouten ${ }^{159 a}$, J. Schovancova ${ }^{125}$, M. Schram ${ }^{85}$, C. Schroeder ${ }^{81}$, N. Schroer ${ }^{58}$, S. Schuh ${ }^{29}$, G. Schuler ${ }^{29}$, J. Schultes ${ }^{174}$, H.-C. Schultz-Coulon ${ }^{58 a}$, H. Schulz ${ }^{15}$, J.W. Schumacher $^{20}$, M. Schumacher ${ }^{48}$, B.A. Schumm ${ }^{137}$, Ph. Schune ${ }^{136}$, C. Schwanenberger ${ }^{82}$, A. Schwartzman ${ }^{143}, \mathrm{Ph}_{\text {. Schwem- }}$ $\operatorname{ling}^{78}$, R. Schwienhorst ${ }^{88}$, R. Schwierz ${ }^{43}$, J. Schwindling ${ }^{136}$, T. Schwindt ${ }^{20}$, M. Schwoerer ${ }^{4}$, W.G. Scott ${ }^{129}$, J. Searcy ${ }^{114}$, G. $\operatorname{Sedov}^{41}$, E. Sedykh ${ }^{121}$, E. Segura ${ }^{11}$, S.C. Seidel ${ }^{103}$, A. Seiden ${ }^{137}$, F. Seifert ${ }^{43}$, J.M. Seixas ${ }^{23 a}$, G. Sekhniaidze ${ }^{102 a}$, D.M. Seliverstov ${ }^{121}$, B. Sellden ${ }^{146 a}$, G. Sellers ${ }^{73}$, M. Seman ${ }^{144 b}$, N. Semprini-Cesari ${ }^{19 a, 19 b}$, C. Serfon ${ }^{98}$, L. Serin ${ }^{115}$, R. Seuster ${ }^{99}$, H. Severini ${ }^{111}$, M.E. Sevior ${ }^{86}$, A. Sfyrla ${ }^{29}$, E. Shabalina ${ }^{54}$, M. Shamim ${ }^{114}$, L.Y. Shan ${ }^{32 a}$, J.T. Shank ${ }^{21}$, Q.T. Shao ${ }^{86}$, M. Shapiro ${ }^{14}$, P.B. Shatalov ${ }^{95}$, L. Shaver ${ }^{6}$, K. Shaw ${ }^{164 a, 164 c}$, D. Sherman ${ }^{175}$, P. Sherwood ${ }^{77}$, A. Shibata ${ }^{108}$, H. Shichi ${ }^{101}$, S. Shimizu ${ }^{29}$, M. Shimojima ${ }^{100}$, T. Shin $^{56}$, M. Shiyakova ${ }^{65}$, A. Shmeleva ${ }^{94}$, M.J. Shochet ${ }^{30}$, D. Short ${ }^{118}$, 
S. Shrestha ${ }^{64}$, M.A. Shupe ${ }^{6}$, P. Sicho ${ }^{125}$, A. Sidoti ${ }^{132 a}$, A. Siebel ${ }^{174}$, F. Siegert ${ }^{48}$, Dj. Sijacki ${ }^{12 a}$, O. Silbert ${ }^{171}$, J. Silva ${ }^{124 a, b}$, Y. Silver ${ }^{153}$, D. Silverstein ${ }^{143}$, S.B. Silverstein ${ }^{146 a}$, V. Simak ${ }^{127}$, O. Simard ${ }^{136}$, Lj. Simic ${ }^{12 a}$, S. Simion ${ }^{115}$, B. Simmons ${ }^{77}$, M. Simonyan ${ }^{35}$, P. Sinervo ${ }^{158}$, N.B. Sinev ${ }^{114}$, V. Sipica ${ }^{141}$, G. Siragusa ${ }^{173}$, A. Sircar ${ }^{24}$, A.N. Sisakyan ${ }^{65}$, S.Yu. Sivoklokov $^{97}$, J. Sjölin ${ }^{146 a, 146 b}$, T.B. Sjursen ${ }^{13}$, L.A. Skinnari ${ }^{14}$, H.P. Skottowe ${ }^{57}$, K. Skovpen ${ }^{107}$, P. Skubic ${ }^{111}$, N. Skvorodnev ${ }^{22}$, M. Slater ${ }^{17}$, T. Slavicek ${ }^{127}$, K. Sliwa ${ }^{161}$, J. Sloper ${ }^{29}$, V. Smakhtin ${ }^{171}$, S.Yu. Smirnov ${ }^{96}$, L.N. Smirnova ${ }^{97}$, O. Smirnova ${ }^{79}$, B.C. Smith ${ }^{57}$, D. Smith ${ }^{143}$, K.M. Smith ${ }^{53}$, M. Smizanska ${ }^{71}$, K. Smolek ${ }^{127}$, A.A. Snesarev ${ }^{94}$, S.W. Snow ${ }^{82}$, J. Snow ${ }^{111}$, J. Snuverink ${ }^{105}$, S. Snyder ${ }^{24}$, M. Soares ${ }^{124 a}$, R. Sobie ${ }^{169, j}$, J. Sodomka ${ }^{127}$, A. Soffer ${ }^{153}$, C.A. Solans ${ }^{167}$, M. Solar ${ }^{127}$, J. Solc ${ }^{127}$, E. Soldatov ${ }^{96}$, U. Soldevila ${ }^{167}$, E. Solfaroli Camillocci ${ }^{132 a, 132 b}$, A.A. Solodkov ${ }^{128}$, O.V. Solovyanov ${ }^{128}$, J. Sondericker $^{24}$, N. Soni ${ }^{2}$, V. Sopko ${ }^{127}$, B. Sopko ${ }^{127}$, M. Sosebee ${ }^{7}$, R. Soualah ${ }^{164 a, 164 c}$, A. Soukharev ${ }^{107}$, S. Spagnolo ${ }^{72 a, 72 b}$, F. Spanò ${ }^{76}$, R. Spighi ${ }^{19 a}$, G. Spigo ${ }^{29}$, F. Spila ${ }^{132 a, 132 b}$, R. Spiwoks ${ }^{29}$, M. Spousta ${ }^{126}$, T. Spreitzer ${ }^{158}$, B. Spurlock ${ }^{7}$, R.D. St. Denis ${ }^{53}$, T. Stahl ${ }^{141}$, J. Stahlman ${ }^{120}$, R. Stamen ${ }^{58 a}$, E. Stanecka ${ }^{38}$, R.W. Stanek ${ }^{5}$, C. Stanescu ${ }^{134 a}$, S. Stapnes ${ }^{117}$, E.A. Starchenko ${ }^{128}$, J. Stark ${ }^{55}$, P. Staroba ${ }^{125}$, P. Starovoitov ${ }^{91}$, A. Staude ${ }^{98}$, P. Stavina ${ }^{144 a}$, G. Stavropoulos ${ }^{14}$, G. Steele ${ }^{53}$, P. Steinbach ${ }^{43}$, P. Steinberg ${ }^{24}$, I. Stek1 ${ }^{127}$, B. Stelzer ${ }^{142}$, H.J. Stelzer ${ }^{88}$, O. Stelzer-Chilton ${ }^{159 a}$, H. Stenzel ${ }^{52}$, S. Stern ${ }^{99}$, K. Stevenson ${ }^{75}$, G.A. Stewart ${ }^{29}$, J.A. Stillings ${ }^{20}$, M.C. Stockton ${ }^{29}$, K. Stoerig ${ }^{48}$, G. Stoicea ${ }^{25 a}$, S. Stonjek ${ }^{99}$, P. Strachota ${ }^{126}$, A.R. Stradling 7 , A. Straessner ${ }^{43}$, J. Strandberg ${ }^{147}$, S. Strandberg ${ }^{146 a, 146 b}$, A. Strandlie ${ }^{117}$, M. Strang ${ }^{109}$, E. Strauss ${ }^{143}$, M. Strauss ${ }^{111}$, P. Strizenec ${ }^{144 b}$, R. Ströhmer ${ }^{173}$, D.M. Strom ${ }^{114}$, J.A. Strong ${ }^{76, *}$, R. Stroynowski ${ }^{39}$, J. Strube ${ }^{129}$, B. Stugu ${ }^{13}$, I. Stumer ${ }^{24, *}$, J. Stupak ${ }^{148}$, P. Sturm ${ }^{174}$, N.A. Styles ${ }^{41}$, D.A. Soh ${ }^{151, u}$, D. Su ${ }^{143}$, HS. Subramania ${ }^{2}$, A. Succurro ${ }^{11}$, Y. Sugaya $^{116}$, T. Sugimoto ${ }^{101}$, C. Suhr ${ }^{106}$, K. Suita ${ }^{67}$, M. Suk ${ }^{126}$, V.V. Sulin ${ }^{94}$, S. Sultansoy ${ }^{3 d}$, T. Sumida ${ }^{68}$, X. Sun ${ }^{55}$, J.E. Sundermann $^{48}$, K. Suruliz ${ }^{139}$, S. Sushkov ${ }^{11}$, G. Susinno ${ }^{36 a, 36 b}$, M.R. Sutton ${ }^{149}$, Y. Suzuki ${ }^{66}$, Y. Suzuki ${ }^{67}$, M. Svatos ${ }^{125}$, Yu.M. Sviri$\operatorname{dov}^{128}$, S. Swedish ${ }^{168}$, I. Sykora ${ }^{144 a}$, T. Sykora ${ }^{126}$, B. Szeless ${ }^{29}$, J. Sánchez ${ }^{167}$, D. Ta ${ }^{105}$, K. Tackmann ${ }^{41}$, A. Taffard ${ }^{163}$, R. Tafirout ${ }^{159}$ a N. Taiblum ${ }^{153}$, Y. Takahashi ${ }^{101}$, H. Takai ${ }^{24}$, R. Takashima ${ }^{69}$, H. Takeda ${ }^{67}$, T. Takeshita ${ }^{140}$, M. Talby $^{83}$, A. Talyshev ${ }^{107, f}$, M.C. Tamsett ${ }^{24}$, J. Tanaka ${ }^{155}$, R. Tanaka ${ }^{115}$, S. Tanaka ${ }^{131}$, S. Tanaka ${ }^{66}$, Y. Tanaka ${ }^{100}$, K. Tani ${ }^{67}$, N. Tannoury $^{83}$, G.P. Tappern ${ }^{29}$, S. Tapprogge ${ }^{81}$, D. Tardif ${ }^{158}$, S. Tarem ${ }^{152}$, F. Tarrade ${ }^{28}$, G.F. Tartarelli ${ }^{89 a}$, P. Tas ${ }^{126}$, M. Tasevsky $^{125}$, E. Tassi ${ }^{36 a, 36 b}$, M. Tatarkhanov ${ }^{14}$, Y. Tayalati ${ }^{135 d}$, C. Taylor ${ }^{77}$, F.E. Taylor ${ }^{92}$, G.N. Taylor ${ }^{86}$, W. Taylor ${ }^{159 b}$, M. Teinturier ${ }^{115}$, M. Teixeira Dias Castanheira ${ }^{75}$, P. Teixeira-Dias ${ }^{76}$, K.K. Temming ${ }^{48}$, H. Ten Kate ${ }^{29}$, P.K. Teng ${ }^{151}$, S. Terada $^{66}$, K. Terashi ${ }^{155}$, J. Terron ${ }^{80}$, M. Testa ${ }^{47}$, R.J. Teuscher ${ }^{158, j}$, J. Thadome ${ }^{174}$, J. Therhaag ${ }^{20}$, T. Theveneaux-Pelzer ${ }^{78}$, M. Thioye ${ }^{175}$, S. Thoma ${ }^{48}$, J.P. Thomas ${ }^{17}$, E.N. Thompson ${ }^{34}$, P.D. Thompson ${ }^{17}$, P.D. Thompson ${ }^{158}$, A.S. Thompson ${ }^{53}$, E. Thomson ${ }^{120}$, M. Thomson ${ }^{27}$, R.P. Thun ${ }^{87}$, F. Tian ${ }^{34}$, M.J. Tibbetts ${ }^{14}$, T. Tic ${ }^{125}$, V.O. Tikhomirov ${ }^{94}$, Y.A. Tikhonov ${ }^{107, f}$, S Timoshenko ${ }^{96}$, P. Tipton ${ }^{175}$, F.J. Tique Aires Viegas ${ }^{29}$, S. Tisserant ${ }^{83}$, B. Toczek ${ }^{37}$, T. Todorov ${ }^{4}$, S. Todorova-Nova ${ }^{161}$, B. Toggerson ${ }^{163}$, J. Tojo ${ }^{66}$, S. Tokár ${ }^{144 a}$, K. Tokunaga ${ }^{67}$, K. Tokushuku ${ }^{66}$, K. Tollefson ${ }^{88}$, M. Tomoto ${ }^{101}$, L. Tompkins $^{30}$, K. Toms ${ }^{103}$, G. Tong ${ }^{32 a}$, A. Tonoyan ${ }^{13}$, C. Topfel ${ }^{16}$, N.D. Topilin ${ }^{65}$, I. Torchiani ${ }^{29}$, E. Torrence ${ }^{114}$, H. Torres ${ }^{78}$, E. Torró Pastor ${ }^{167}$, J. Toth ${ }^{83 \text {,aa }, \text { F. Touchard }}{ }^{83}$, D.R. Tovey ${ }^{139}$, T. Trefzger ${ }^{173}$, L. Tremblet ${ }^{29}$, A. Tricoli ${ }^{29}$, I.M. Trigger ${ }^{159 a}$, S. Trincaz-Duvoid ${ }^{78}$, T.N. Trinh ${ }^{78}$, M.F. Tripiana ${ }^{70}$, W. Trischuk ${ }^{158}$, A. Trivedii ${ }^{24, z}$, B. Trocmé ${ }^{55}$, C. Troncon ${ }^{89 a}$, M. TrottierMcDonald $^{142}$, M. Trzebinski ${ }^{38}$, A. Trzupek ${ }^{38}$, C. Tsarouchas ${ }^{29}$, J.C-L. Tseng ${ }^{118}$, M. Tsiakiris ${ }^{105}$, P.V. Tsiareshka ${ }^{90}$, D. Tsionou ${ }^{4, a e}$, G. Tsipolitis $^{9}$, V. Tsiskaridze ${ }^{48}$, E.G. Tskhadadze ${ }^{51 a}$, I.I. Tsukerman ${ }^{95}$, V. Tsulaia ${ }^{14}$, J.-W. Tsung ${ }^{20}$,

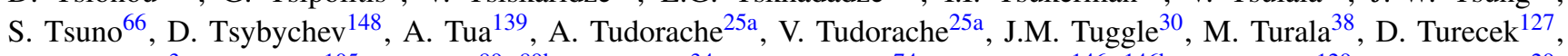

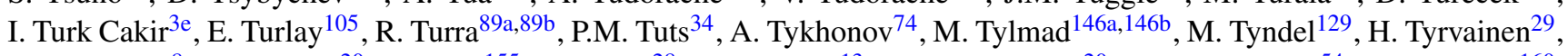
G. Tzanakos $^{8}$, K. Uchida ${ }^{20}$, I. Ueda ${ }^{155}$, R. Ueno ${ }^{28}$, M. Ugland ${ }^{13}$, M. Uhlenbrock ${ }^{20}$, M. Uhrmacher ${ }^{54}$, F. Ukegawa ${ }^{160}$, G. Unal ${ }^{29}$, D.G. Underwood ${ }^{5}$, A. Undrus ${ }^{24}$, G. Unel ${ }^{163}$, Y. Unno ${ }^{66}$, D. Urbaniec ${ }^{34}$, G. Usai ${ }^{7}$, M. Uslenghi ${ }^{119 a, 119 b}$, L. Vacavant $^{83}$, V. Vacek ${ }^{127}$, B. Vachon ${ }^{85}$, S. Vahsen ${ }^{14}$, J. Valenta ${ }^{125}$, P. Valente ${ }^{132 a}$, S. Valentinetti19a,19b, S. Valkar ${ }^{126}$, E. Valladolid Gallego ${ }^{167}$, S. Vallecorsa ${ }^{152}$, J.A. Valls Ferrer ${ }^{167}$, H. van der Graaf ${ }^{105}$, E. van der Kraaij ${ }^{105}$, R. Van Der Leeuw ${ }^{105}$, E. van der Poel ${ }^{105}$, D. van der $\operatorname{Ster}^{29}$, N. van $\operatorname{Eldik}^{84}$, P. van Gemmeren ${ }^{5}$, Z. van Kesteren ${ }^{105}$, I. van Vulpen ${ }^{105}$, M. Vanadia $^{99}$, W. Vandelli ${ }^{29}$, G. Vandoni ${ }^{29}$, A. Vaniachine ${ }^{5}$, P. Vankov ${ }^{41}$, F. Vannucci ${ }^{78}$, F. Varela Rodriguez ${ }^{29}$, R. Vari ${ }^{132 a}$, E.W. Varnes ${ }^{6}$, D. Varouchas ${ }^{14}$, A. Vartapetian ${ }^{7}$, K.E. Varvel1 ${ }^{150}$, V.I. Vassilakopoulos ${ }^{56}$, F. Vazeille ${ }^{33}$, G. Vegni ${ }^{89 a, 89 b}$,

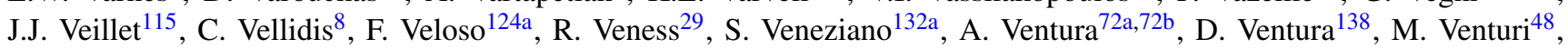
N. Venturi ${ }^{158}$, V. Vercesi ${ }^{119 a}$, M. Verducci ${ }^{138}$, W. Verkerke ${ }^{105}$, J.C. Vermeulen ${ }^{105}$, A. Vest ${ }^{43}$, M.C. Vetterli ${ }^{142, d}$, I. Vichou $^{165}$, T. Vickey ${ }^{145 b \text {,af }}$, O.E. Vickey Boeriu ${ }^{145 b}$, G.H.A. Viehhauser ${ }^{118}$, S. Viel $^{168}$, M. Villa ${ }^{19 a, 19 b}$, M. Villaplana Perez ${ }^{167}$, E. Vilucchi ${ }^{47}$, M.G. Vincter ${ }^{28}$, E. Vinek ${ }^{29}$, V.B. Vinogradov ${ }^{65}$, M. Virchaux ${ }^{136, *}$, J. Virzi ${ }^{14}$, O. Vitells ${ }^{171}$, M. Viti ${ }^{41}$, I. Vi-

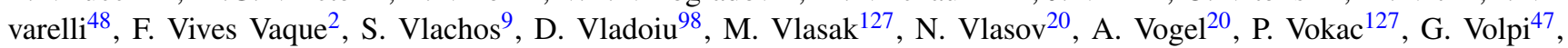
M. Volpi ${ }^{86}$, G. Volpini ${ }^{89 a}$, H. von der Schmitt ${ }^{99}$, J. von Loeben ${ }^{99}$, H. von Radziewski ${ }^{48}$, E. von Toerne ${ }^{20}$, V. Vorobel $^{126}$, A.P. Vorobiev ${ }^{128}$, V. Vorwerk ${ }^{11}$, M. Vos $^{167}$, R. Voss $^{29}$, T.T. $\operatorname{Voss}^{174}$, J.H. Vossebeld ${ }^{73}$, N. Vranjes ${ }^{12 a}$, M. Vranjes Milosavljevic $^{105}$, V. Vrba ${ }^{125}$, M. Vreeswijk ${ }^{105}$, T. Vu $\mathrm{Anh}^{81}$, R. Vuillermet ${ }^{29}$, I. Vukotic ${ }^{115}$, W. Wagner ${ }^{174}$, P. Wagner ${ }^{120}$, H. Wahlen ${ }^{174}$, J. Wakabayashi ${ }^{101}$, J. Walbersloh ${ }^{42}$, S. Walch ${ }^{87}$, J. Walder ${ }^{71}$, R. Walker ${ }^{98}$, W. Walkowiak ${ }^{141}$, R. Wall ${ }^{175}$, 
P. Waller ${ }^{73}$, C. Wang ${ }^{44}$, H. Wang ${ }^{172}$, H. Wang ${ }^{32 b, a g}$, J. Wang ${ }^{151}$, J. Wang ${ }^{55}$, J.C. Wang ${ }^{138}$, R. Wang ${ }^{103}$, S.M. Wang ${ }^{151}$, A. Warburton ${ }^{85}$, C.P. Ward ${ }^{27}$, M. Warsinsky ${ }^{48}$, R. Wastie ${ }^{118}$, P.M. Watkins ${ }^{17}$, A.T. Watson ${ }^{17}$, I.J. Watson ${ }^{150}$, M.F. Wat$\operatorname{son}^{17}$, G. Watts ${ }^{138}$, S. Watts ${ }^{82}$, A.T. Waugh ${ }^{150}$, B.M. Waugh ${ }^{77}$, J. Weber ${ }^{42}$, M. Weber ${ }^{129}$, M.S. Weber ${ }^{16}$, P. Weber ${ }^{54}$, A.R. Weidberg ${ }^{118}$, P. Weigell ${ }^{99}$, J. Weingarten ${ }^{54}$, C. Weiser ${ }^{48}$, H. Wellenstein ${ }^{22}$, P.S. Wells ${ }^{29}$, M. Wen ${ }^{47}$, T. Wenaus ${ }^{24}$, S. Wendler ${ }^{123}$, Z. Weng ${ }^{151, \text { u }}$, T. Wengler ${ }^{29}$, S. Wenig ${ }^{29}$, N. Wermes ${ }^{20}$, M. Werner $^{48}$, P. Werner $^{29}$, M. Werth ${ }^{163}$, M. Wessels $^{58 a}$, C. Weydert ${ }^{55}$, K. Whalen ${ }^{28}$, S.J. Wheeler-Ellis ${ }^{163}$, S.P. Whitaker ${ }^{21}$, A. White ${ }^{7}$, M.J. White ${ }^{86}$, S.R. Whitehead ${ }^{118}$, D. Whiteson $^{163}$, D. Whittington ${ }^{61}$, F. Wicek ${ }^{115}$, D. Wicke ${ }^{174}$, F.J. Wickens ${ }^{129}$, W. Wiedenmann ${ }^{172}$, M. Wielers ${ }^{129}$, P. Wienemann ${ }^{20}$, C. Wiglesworth ${ }^{75}$, L.A.M. Wiik-Fuchs ${ }^{48}$, P.A. Wijeratne ${ }^{77}$, A. Wildauer ${ }^{167}$, M.A. Wildt ${ }^{41, \mathrm{q}}$, I. Wilhelm ${ }^{126}$, H.G. Wilkens ${ }^{29}$, J.Z. Will ${ }^{98}$, E. Williams ${ }^{34}$, H.H. Williams ${ }^{120}$, W. Willis ${ }^{34}$, S. Willocq ${ }^{84}$, J.A. Wilson ${ }^{17}$, M.G. Wilson ${ }^{143}$, A. Wilson ${ }^{87}$, I. Wingerter-Seez ${ }^{4}$, S. Winkelmann ${ }^{48}$, F. Winklmeier ${ }^{29}$, M. Wittgen ${ }^{143}$, M.W. Wolter ${ }^{38}$, H. Wolters ${ }^{124 a, h}$, W.C. Wong ${ }^{40}$, G. Wooden ${ }^{87}$, B.K. Wosiek ${ }^{38}$, J. Wotschack ${ }^{29}$, M.J. Woudstra ${ }^{84}$, K.W. Wozniak ${ }^{38}$, K. Wraight ${ }^{53}$, C. Wright ${ }^{53}$, M. Wright ${ }^{53}$, B. Wrona ${ }^{73}$, S.L. $\mathrm{Wu}^{172}$, X. Wu ${ }^{49}$, Y. Wu ${ }^{32 b \text {,ah }, \text { E. Wulf }}{ }^{34}$, R. Wunstorf ${ }^{42}$, B.M. Wynne ${ }^{45}$, S. Xella ${ }^{35}$, M. Xiao ${ }^{136}$, S. Xie $^{48}$, Y. Xie ${ }^{32 a}$, C. Xu ${ }^{32 b, w}$, D. Xu ${ }^{139}$, G. Xu $^{32 a}$, B. Yabsley ${ }^{150}$, S. Yacoob ${ }^{145 b}$, M. Yamada ${ }^{66}$, H. Yamaguchi ${ }^{155}$, A. Yamamoto ${ }^{66}$, K. Yamamoto ${ }^{64}$, S. Yamamoto ${ }^{155}$, T. Yamamura ${ }^{155}$, T. Yamanaka ${ }^{155}$, J. Yamaoka ${ }^{44}$, T. Yamazaki ${ }^{155}$, Y. Yamazaki ${ }^{67}$,

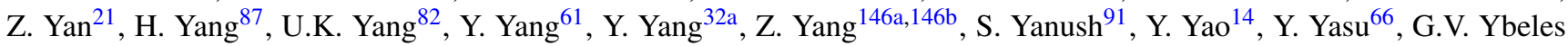

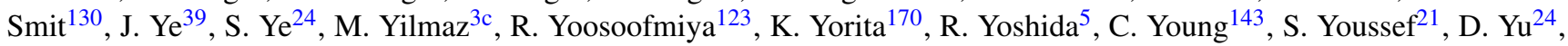
J. Yu ${ }^{7}$, J. Yu ${ }^{112}$, L. Yuan ${ }^{32 a, a i}$, A. Yurkewicz ${ }^{106}$, B. Zabinski ${ }^{38}$, V.G. Zaets ${ }^{128}$, R. Zaidan ${ }^{63}$, A.M. Zaitsev ${ }^{128}$, Z. Zajacova ${ }^{29}$, Yo.K. Zalite ${ }^{121}$, L. Zanello ${ }^{132 a, 132 b}$, P. Zarzhitsky ${ }^{39}$, A. Zaytsev ${ }^{107}$, C. Zeitnitz ${ }^{174}$, M. Zeller ${ }^{175}$, M. Zeman ${ }^{125}$, A. Zemla ${ }^{38}$, C. Zendler ${ }^{20}$, O. Zenin ${ }^{128}$, T. Ženišs ${ }^{144 a}$, Z. Zinonos ${ }^{122 a, 122 b}$, S. Zenz ${ }^{14}$, D. Zerwas ${ }^{115}$, G. Zevi della Porta ${ }^{57}$, Z. Zhan ${ }^{32 d}$, D. Zhang ${ }^{32 b, a g}$, H. Zhang ${ }^{88}$, J. Zhang ${ }^{5}$, X. Zhang ${ }^{32 d}$, Z. Zhang ${ }^{115}$, L. Zhao ${ }^{108}$, T. Zhao ${ }^{138}$, Z. Zhao ${ }^{32 b}$, A. Zhemchugov ${ }^{65}$, S. Zheng ${ }^{32 a}$, J. Zhong ${ }^{118}$, B. Zhou ${ }^{87}$, N. Zhou ${ }^{163}$, Y. Zhou ${ }^{151}$, C.G. Zhu ${ }^{32 d}$, H. Zhu ${ }^{41}$, J. Zhu ${ }^{87}$, Y. Zhu ${ }^{32 b}$, X. Zhuang ${ }^{98}$, V. Zhuravlov ${ }^{99}$, D. Zieminska ${ }^{61}$, R. Zimmermann ${ }^{20}$, S. Zimmermann ${ }^{20}$, S. Zimmermann ${ }^{48}$, M. Ziolkowski ${ }^{141}$, R. Zitoun ${ }^{4}$, L. Živković ${ }^{34}$, V.V. Zmouchko ${ }^{128, *}$, G. Zobernig ${ }^{172}$, A. Zoccoli ${ }^{19 a, 19 b}$, Y. Zolnierowski ${ }^{4}$, A. Zsenei ${ }^{29}$, M. zur Nedden ${ }^{15}$, V. Zutshi ${ }^{106}$, L. Zwalinski ${ }^{29}$

${ }^{1}$ University at Albany, Albany NY, United States of America

${ }^{2}$ Department of Physics, University of Alberta, Edmonton AB, Canada

${ }^{3(a)}$ Department of Physics, Ankara University, Ankara; ${ }^{(b)}$ Department of Physics, Dumlupinar University, Kutahya;

(c) Department of Physics, Gazi University, Ankara; ${ }^{(d)}$ Division of Physics, TOBB University of Economics and

Technology, Ankara; ${ }^{(e)}$ Turkish Atomic Energy Authority, Ankara, Turkey

${ }^{4}$ LAPP, CNRS/IN2P3 and Université de Savoie, Annecy-le-Vieux, France

${ }^{5}$ High Energy Physics Division, Argonne National Laboratory, Argonne IL, United States of America

${ }^{6}$ Department of Physics, University of Arizona, Tucson AZ, United States of America

${ }^{7}$ Department of Physics, The University of Texas at Arlington, Arlington TX, United States of America

${ }^{8}$ Physics Department, University of Athens, Athens, Greece

${ }^{9}$ Physics Department, National Technical University of Athens, Zografou, Greece

${ }^{10}$ Institute of Physics, Azerbaijan Academy of Sciences, Baku, Azerbaijan

${ }^{11}$ Institut de Física d'Altes Energies and Departament de Física de la Universitat Autònoma de Barcelona and ICREA,

Barcelona, Spain

${ }^{12(a)}$ Institute of Physics, University of Belgrade, Belgrade; ${ }^{(b)}$ Vinca Institute of Nuclear Sciences, University of Belgrade,

Belgrade, Serbia

${ }^{13}$ Department for Physics and Technology, University of Bergen, Bergen, Norway

${ }^{14}$ Physics Division, Lawrence Berkeley National Laboratory and University of California, Berkeley CA, United States of America

${ }^{15}$ Department of Physics, Humboldt University, Berlin, Germany

${ }^{16}$ Albert Einstein Center for Fundamental Physics and Laboratory for High Energy Physics, University of Bern, Bern, Switzerland

${ }^{17}$ School of Physics and Astronomy, University of Birmingham, Birmingham, United Kingdom

${ }^{18(a)}$ Department of Physics, Bogazici University, Istanbul; ${ }^{(b)}$ Division of Physics, Dogus University, Istanbul;

${ }^{(c)}$ Department of Physics Engineering, Gaziantep University, Gaziantep; ${ }^{(d)}$ Department of Physics, Istanbul Technical University, Istanbul, Turkey

19(a) INFN Sezione di Bologna; ${ }^{(b)}$ Dipartimento di Fisica, Università di Bologna, Bologna, Italy

${ }^{20}$ Physikalisches Institut, University of Bonn, Bonn, Germany

${ }^{21}$ Department of Physics, Boston University, Boston MA, United States of America 
${ }^{22}$ Department of Physics, Brandeis University, Waltham MA, United States of America

${ }^{23(a)}$ Universidade Federal do Rio De Janeiro COPPE/EE/IF, Rio de Janeiro; ${ }^{\left({ }^{b}\right)}$ Federal University of Juiz de Fora (UFJF),

Juiz de Fora; ${ }^{(c)}$ Federal University of Sao Joao del Rei (UFSJ), Sao Joao del Rei; ${ }^{(d)}$ Instituto de Fisica, Universidade de

Sao Paulo, Sao Paulo, Brazil

${ }^{24}$ Physics Department, Brookhaven National Laboratory, Upton NY, United States of America

${ }^{25(a)}$ National Institute of Physics and Nuclear Engineering, Bucharest; ${ }^{(b)}$ University Politehnica Bucharest, Bucharest;

${ }^{(c)}$ West University in Timisoara, Timisoara, Romania

${ }^{26}$ Departamento de Física, Universidad de Buenos Aires, Buenos Aires, Argentina

${ }^{27}$ Cavendish Laboratory, University of Cambridge, Cambridge, United Kingdom

${ }^{28}$ Department of Physics, Carleton University, Ottawa ON, Canada

${ }^{29}$ CERN, Geneva, Switzerland

${ }^{30}$ Enrico Fermi Institute, University of Chicago, Chicago IL, United States of America

31(a) Departamento de Fisica, Pontificia Universidad Católica de Chile, Santiago; ${ }^{(b)}$ Departamento de Física, Universidad

Técnica Federico Santa María, Valparaíso, Chile

${ }^{32(a)}$ Institute of High Energy Physics, Chinese Academy of Sciences, Beijing; ${ }^{(b)}$ Department of Modern Physics,

University of Science and Technology of China, Anhui; ${ }^{(c)}$ Department of Physics, Nanjing University, Jiangsu; ${ }^{(d)}$ School of Physics, Shandong University, Shandong, China

${ }^{33}$ Laboratoire de Physique Corpusculaire, Clermont Université and Université Blaise Pascal and CNRS/IN2P3, Aubiere Cedex, France

${ }^{34}$ Nevis Laboratory, Columbia University, Irvington NY, United States of America

${ }^{35}$ Niels Bohr Institute, University of Copenhagen, Kobenhavn, Denmark

36(a) INFN Gruppo Collegato di Cosenza; ${ }^{(b)}$ Dipartimento di Fisica, Università della Calabria, Arcavata di Rende, Italy

${ }^{37}$ Faculty of Physics and Applied Computer Science, AGH University of Science and Technology, Krakow, Poland

${ }^{38}$ The Henryk Niewodniczanski Institute of Nuclear Physics, Polish Academy of Sciences, Krakow, Poland

${ }^{39}$ Physics Department, Southern Methodist University, Dallas TX, United States of America

${ }^{40}$ Physics Department, University of Texas at Dallas, Richardson TX, United States of America

${ }^{41}$ DESY, Hamburg and Zeuthen, Germany

${ }^{42}$ Institut für Experimentelle Physik IV, Technische Universität Dortmund, Dortmund, Germany

${ }^{43}$ Institut für Kern- und Teilchenphysik, Technical University Dresden, Dresden, Germany

${ }^{44}$ Department of Physics, Duke University, Durham NC, United States of America

${ }^{45}$ SUPA - School of Physics and Astronomy, University of Edinburgh, Edinburgh, United Kingdom

${ }^{46}$ Fachhochschule Wiener Neustadt, Johannes Gutenbergstrasse 3, 2700 Wiener Neustadt, Austria

${ }^{47}$ INFN Laboratori Nazionali di Frascati, Frascati, Italy

${ }^{48}$ Fakultät für Mathematik und Physik, Albert-Ludwigs-Universität, Freiburg i.Br., Germany

${ }^{49}$ Section de Physique, Université de Genève, Geneva, Switzerland

50(a) INFN Sezione di Genova; ${ }^{(b)}$ Dipartimento di Fisica, Università di Genova, Genova, Italy

${ }^{51(a)}$ E.Andronikashvili Institute of Physics, Tbilisi State University, Tbilisi; ${ }^{(b)}$ High Energy Physics Institute, Tbilisi State

University, Tbilisi, Georgia

${ }^{52}$ II Physikalisches Institut, Justus-Liebig-Universität Giessen, Giessen, Germany

${ }^{53}$ SUPA - School of Physics and Astronomy, University of Glasgow, Glasgow, United Kingdom

${ }^{54}$ II Physikalisches Institut, Georg-August-Universität, Göttingen, Germany

${ }^{55}$ Laboratoire de Physique Subatomique et de Cosmologie, Université Joseph Fourier and CNRS/IN2P3 and Institut

National Polytechnique de Grenoble, Grenoble, France

${ }^{56}$ Department of Physics, Hampton University, Hampton VA, United States of America

${ }^{57}$ Laboratory for Particle Physics and Cosmology, Harvard University, Cambridge MA, United States of America

58(a) Kirchhoff-Institut für Physik, Ruprecht-Karls-Universität Heidelberg, Heidelberg; (b) Physikalisches Institut,

Ruprecht-Karls-Universität Heidelberg, Heidelberg; ${ }^{(c)}$ ZITI Institut für technische Informatik,

Ruprecht-Karls-Universität Heidelberg, Mannheim, Germany

${ }^{59}$ Faculty of Science, Hiroshima University, Hiroshima, Japan

${ }^{60}$ Faculty of Applied Information Science, Hiroshima Institute of Technology, Hiroshima, Japan

${ }^{61}$ Department of Physics, Indiana University, Bloomington IN, United States of America

${ }^{62}$ Institut für Astro- und Teilchenphysik, Leopold-Franzens-Universität, Innsbruck, Austria

${ }^{63}$ University of Iowa, Iowa City IA, United States of America 
${ }^{64}$ Department of Physics and Astronomy, Iowa State University, Ames IA, United States of America

${ }^{65}$ Joint Institute for Nuclear Research, JINR Dubna, Dubna, Russia

${ }^{66}$ KEK, High Energy Accelerator Research Organization, Tsukuba, Japan

${ }^{67}$ Graduate School of Science, Kobe University, Kobe, Japan

${ }^{68}$ Faculty of Science, Kyoto University, Kyoto, Japan

${ }^{69}$ Kyoto University of Education, Kyoto, Japan

${ }^{70}$ Instituto de Física La Plata, Universidad Nacional de La Plata and CONICET, La Plata, Argentina

${ }^{71}$ Physics Department, Lancaster University, Lancaster, United Kingdom

72(a) INFN Sezione di Lecce; ${ }^{(b)}$ Dipartimento di Fisica, Università del Salento, Lecce, Italy

${ }^{73}$ Oliver Lodge Laboratory, University of Liverpool, Liverpool, United Kingdom

${ }^{74}$ Department of Physics, Jožef Stefan Institute and University of Ljubljana, Ljubljana, Slovenia

${ }^{75}$ School of Physics and Astronomy, Queen Mary University of London, London, United Kingdom

${ }^{76}$ Department of Physics, Royal Holloway University of London, Surrey, United Kingdom

${ }^{77}$ Department of Physics and Astronomy, University College London, London, United Kingdom

${ }^{78}$ Laboratoire de Physique Nucléaire et de Hautes Energies, UPMC and Université Paris-Diderot and CNRS/IN2P3, Paris, France

${ }^{79}$ Fysiska institutionen, Lunds universitet, Lund, Sweden

${ }^{80}$ Departamento de Fisica Teorica C-15, Universidad Autonoma de Madrid, Madrid, Spain

${ }^{81}$ Institut für Physik, Universität Mainz, Mainz, Germany

${ }^{82}$ School of Physics and Astronomy, University of Manchester, Manchester, United Kingdom

${ }^{83}$ CPPM, Aix-Marseille Université and CNRS/IN2P3, Marseille, France

${ }^{84}$ Department of Physics, University of Massachusetts, Amherst MA, United States of America

${ }^{85}$ Department of Physics, McGill University, Montreal QC, Canada

${ }^{86}$ School of Physics, University of Melbourne, Victoria, Australia

${ }^{87}$ Department of Physics, The University of Michigan, Ann Arbor MI, United States of America

${ }^{88}$ Department of Physics and Astronomy, Michigan State University, East Lansing MI, United States of America

89(a) INFN Sezione di Milano; ${ }^{(b)}$ Dipartimento di Fisica, Università di Milano, Milano, Italy

${ }^{90}$ B.I. Stepanov Institute of Physics, National Academy of Sciences of Belarus, Minsk, Republic of Belarus

${ }^{91}$ National Scientific and Educational Centre for Particle and High Energy Physics, Minsk, Republic of Belarus

${ }^{92}$ Department of Physics, Massachusetts Institute of Technology, Cambridge MA, United States of America

${ }^{93}$ Group of Particle Physics, University of Montreal, Montreal QC, Canada

${ }^{94}$ P.N. Lebedev Institute of Physics, Academy of Sciences, Moscow, Russia

${ }^{95}$ Institute for Theoretical and Experimental Physics (ITEP), Moscow, Russia

${ }^{96}$ Moscow Engineering and Physics Institute (MEPhI), Moscow, Russia

${ }^{97}$ Skobeltsyn Institute of Nuclear Physics, Lomonosov Moscow State University, Moscow, Russia

${ }^{98}$ Fakultät für Physik, Ludwig-Maximilians-Universität München, München, Germany

${ }^{99}$ Max-Planck-Institut für Physik (Werner-Heisenberg-Institut), München, Germany

${ }^{100}$ Nagasaki Institute of Applied Science, Nagasaki, Japan

${ }^{101}$ Graduate School of Science, Nagoya University, Nagoya, Japan

102(a) INFN Sezione di Napoli; ${ }^{(b)}$ Dipartimento di Scienze Fisiche, Università di Napoli, Napoli, Italy

${ }^{103}$ Department of Physics and Astronomy, University of New Mexico, Albuquerque NM, United States of America

${ }^{104}$ Institute for Mathematics, Astrophysics and Particle Physics, Radboud University Nijmegen/Nikhef, Nijmegen,

Netherlands

${ }^{105}$ Nikhef National Institute for Subatomic Physics and University of Amsterdam, Amsterdam, Netherlands

${ }^{106}$ Department of Physics, Northern Illinois University, DeKalb IL, United States of America

${ }^{107}$ Budker Institute of Nuclear Physics, SB RAS, Novosibirsk, Russia

${ }^{108}$ Department of Physics, New York University, New York NY, United States of America

${ }^{109}$ Ohio State University, Columbus OH, United States of America

${ }^{110}$ Faculty of Science, Okayama University, Okayama, Japan

${ }^{111}$ Homer L. Dodge Department of Physics and Astronomy, University of Oklahoma, Norman OK, United States of America

${ }^{112}$ Department of Physics, Oklahoma State University, Stillwater OK, United States of America

${ }^{113}$ Palacký University, RCPTM, Olomouc, Czech Republic 
${ }^{114}$ Center for High Energy Physics, University of Oregon, Eugene OR, United States of America

${ }^{115}$ LAL, Univ. Paris-Sud and CNRS/IN2P3, Orsay, France

${ }^{116}$ Graduate School of Science, Osaka University, Osaka, Japan

${ }^{117}$ Department of Physics, University of Oslo, Oslo, Norway

${ }^{118}$ Department of Physics, Oxford University, Oxford, United Kingdom

119(a) INFN Sezione di Pavia; ${ }^{(b)}$ Dipartimento di Fisica, Università di Pavia, Pavia, Italy

${ }^{120}$ Department of Physics, University of Pennsylvania, Philadelphia PA, United States of America

${ }^{121}$ Petersburg Nuclear Physics Institute, Gatchina, Russia

${ }^{122(a)}$ INFN Sezione di Pisa; ${ }^{(b)}$ Dipartimento di Fisica E. Fermi, Università di Pisa, Pisa, Italy

${ }^{123}$ Department of Physics and Astronomy, University of Pittsburgh, Pittsburgh PA, United States of America

${ }^{124(a)}$ Laboratorio de Instrumentacao e Fisica Experimental de Particulas - LIP, Lisboa, Portugal; ${ }^{(b)}$ Departamento de Fisica

Teorica y del Cosmos and CAFPE, Universidad de Granada, Granada, Spain

${ }^{125}$ Institute of Physics, Academy of Sciences of the Czech Republic, Praha, Czech Republic

${ }^{126}$ Faculty of Mathematics and Physics, Charles University in Prague, Praha, Czech Republic

${ }^{127}$ Czech Technical University in Prague, Praha, Czech Republic

${ }^{128}$ State Research Center Institute for High Energy Physics, Protvino, Russia

${ }^{129}$ Particle Physics Department, Rutherford Appleton Laboratory, Didcot, United Kingdom

${ }^{130}$ Physics Department, University of Regina, Regina SK, Canada

${ }^{131}$ Ritsumeikan University, Kusatsu, Shiga, Japan

132(a) INFN Sezione di Roma I; ${ }^{(b)}$ Dipartimento di Fisica, Università La Sapienza, Roma, Italy

133(a) INFN Sezione di Roma Tor Vergata; ${ }^{(b)}$ Dipartimento di Fisica, Università di Roma Tor Vergata, Roma, Italy

134(a) INFN Sezione di Roma Tre; ${ }^{(b)}$ Dipartimento di Fisica, Università Roma Tre, Roma, Italy

${ }^{135(a)}$ Faculté des Sciences Ain Chock, Réseau Universitaire de Physique des Hautes Energies - Université Hassan II,

Casablanca; ${ }^{\left({ }^{b}\right)}$ Centre National de l'Energie des Sciences Techniques Nucleaires, Rabat; ${ }^{(c)}$ Faculté des Sciences

Semlalia, Université Cadi Ayyad, LPHEA-Marrakech; ${ }^{(d)}$ Faculté des Sciences, Université Mohamed Premier and

LPTPM, Oujda; ${ }^{(e)}$ Faculté des Sciences, Université Mohammed V-Agdal, Rabat, Morocco

${ }^{136}$ DSM/IRFU (Institut de Recherches sur les Lois Fondamentales de l'Univers), CEA Saclay (Commissariat a l'Energie Atomique), Gif-sur-Yvette, France

${ }^{137}$ Santa Cruz Institute for Particle Physics, University of California Santa Cruz, Santa Cruz CA, United States of America

${ }^{138}$ Department of Physics, University of Washington, Seattle WA, United States of America

${ }^{139}$ Department of Physics and Astronomy, University of Sheffield, Sheffield, United Kingdom

${ }^{140}$ Department of Physics, Shinshu University, Nagano, Japan

${ }^{141}$ Fachbereich Physik, Universität Siegen, Siegen, Germany

${ }^{142}$ Department of Physics, Simon Fraser University, Burnaby BC, Canada

${ }^{143}$ SLAC National Accelerator Laboratory, Stanford CA, United States of America

${ }^{144(a)}$ Faculty of Mathematics, Physics \& Informatics, Comenius University, Bratislava; (b) Department of Subnuclear

Physics, Institute of Experimental Physics of the Slovak Academy of Sciences, Kosice, Slovak Republic

${ }^{145(\text { a) }}$ Department of Physics, University of Johannesburg, Johannesburg; ${ }^{\left({ }^{b}\right)}$ School of Physics, University of the

Witwatersrand, Johannesburg, South Africa

146(a) Department of Physics, Stockholm University; ${ }^{(b)}$ The Oskar Klein Centre, Stockholm, Sweden

${ }^{147}$ Physics Department, Royal Institute of Technology, Stockholm, Sweden

${ }^{148}$ Departments of Physics \& Astronomy and Chemistry, Stony Brook University, Stony Brook NY, United States of

America

${ }^{149}$ Department of Physics and Astronomy, University of Sussex, Brighton, United Kingdom

${ }^{150}$ School of Physics, University of Sydney, Sydney, Australia

${ }^{151}$ Institute of Physics, Academia Sinica, Taipei, Taiwan

${ }^{152}$ Department of Physics, Technion: Israel Inst. of Technology, Haifa, Israel

${ }^{153}$ Raymond and Beverly Sackler School of Physics and Astronomy, Tel Aviv University, Tel Aviv, Israel

${ }^{154}$ Department of Physics, Aristotle University of Thessaloniki, Thessaloniki, Greece

${ }^{155}$ International Center for Elementary Particle Physics and Department of Physics, The University of Tokyo, Tokyo, Japan

${ }^{156}$ Graduate School of Science and Technology, Tokyo Metropolitan University, Tokyo, Japan

${ }^{157}$ Department of Physics, Tokyo Institute of Technology, Tokyo, Japan

${ }^{158}$ Department of Physics, University of Toronto, Toronto ON, Canada 
159(a) TRIUMF, Vancouver BC; ${ }^{(b)}$ Department of Physics and Astronomy, York University, Toronto ON, Canada

${ }^{160}$ Institute of Pure and Applied Sciences, University of Tsukuba, 1-1-1 Tennodai, Tsukuba, Ibaraki 305-8571, Japan

${ }^{161}$ Science and Technology Center, Tufts University, Medford MA, United States of America

${ }^{162}$ Centro de Investigaciones, Universidad Antonio Narino, Bogota, Colombia

${ }^{163}$ Department of Physics and Astronomy, University of California Irvine, Irvine CA, United States of America

${ }^{164(a)}$ INFN Gruppo Collegato di Udine, Udine; ${ }^{(b)}$ ICTP, Trieste; ${ }^{(c)}$ Dipartimento di Chimica, Fisica e Ambiente, Università di Udine, Udine, Italy

${ }^{165}$ Department of Physics, University of Illinois, Urbana IL, United States of America

${ }^{166}$ Department of Physics and Astronomy, University of Uppsala, Uppsala, Sweden

${ }^{167}$ Instituto de Física Corpuscular (IFIC) and Departamento de Física Atómica, Molecular y Nuclear and Departamento de Ingeniería Electrónica and Instituto de Microelectrónica de Barcelona (IMB-CNM), University of Valencia and CSIC, Valencia, Spain

${ }^{168}$ Department of Physics, University of British Columbia, Vancouver BC, Canada

${ }^{169}$ Department of Physics and Astronomy, University of Victoria, Victoria BC, Canada

${ }^{170}$ Waseda University, Tokyo, Japan

${ }^{171}$ Department of Particle Physics, The Weizmann Institute of Science, Rehovot, Israel

${ }^{172}$ Department of Physics, University of Wisconsin, Madison WI, United States of America

${ }^{173}$ Fakultät für Physik und Astronomie, Julius-Maximilians-Universität, Würzburg, Germany

${ }^{174}$ Fachbereich C Physik, Bergische Universität Wuppertal, Wuppertal, Germany

${ }^{175}$ Department of Physics, Yale University, New Haven CT, United States of America

${ }^{176}$ Yerevan Physics Institute, Yerevan, Armenia

${ }^{177}$ Domaine scientifique de la Doua, Centre de Calcul CNRS/IN2P3, Villeurbanne Cedex, France

${ }^{a}$ Also at Laboratorio de Instrumentacao e Fisica Experimental de Particulas - LIP, Lisboa, Portugal

${ }^{b}$ Also at Faculdade de Ciencias and CFNUL, Universidade de Lisboa, Lisboa, Portugal

${ }^{\mathrm{c}}$ Also at Particle Physics Department, Rutherford Appleton Laboratory, Didcot, United Kingdom

${ }^{\mathrm{d} A l s o}$ at TRIUMF, Vancouver BC, Canada

${ }^{\mathrm{e}}$ Also at Department of Physics, California State University, Fresno CA, United States of America

${ }^{\mathrm{f}}$ Also at Novosibirsk State University, Novosibirsk, Russia

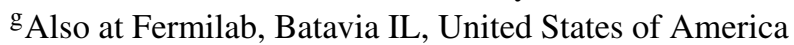

hAlso at Department of Physics, University of Coimbra, Coimbra, Portugal

${ }^{\mathrm{i}}$ Also at Università di Napoli Parthenope, Napoli, Italy

${ }^{\mathrm{j}}$ Also at Institute of Particle Physics (IPP), Canada

${ }^{\mathrm{k}}$ Also at Department of Physics, Middle East Technical University, Ankara, Turkey

${ }^{1}$ Also at Louisiana Tech University, Ruston LA, United States of America

${ }^{m}$ Also at Department of Physics and Astronomy, University College London, London, United Kingdom

${ }^{\mathrm{n}}$ Also at Group of Particle Physics, University of Montreal, Montreal QC, Canada

${ }^{\circ}$ Also at Department of Physics, University of Cape Town, Cape Town, South Africa

${ }^{\mathrm{p}}$ Also at Institute of Physics, Azerbaijan Academy of Sciences, Baku, Azerbaijan

${ }^{\mathrm{q}}$ Also at Institut für Experimentalphysik, Universität Hamburg, Hamburg, Germany

${ }^{\mathrm{r}}$ Also at Manhattan College, New York NY, United States of America

${ }^{\mathrm{s}}$ Also at School of Physics, Shandong University, Shandong, China

${ }^{t}$ Also at CPPM, Aix-Marseille Université and CNRS/IN2P3, Marseille, France

"Also at School of Physics and Engineering, Sun Yat-sen University, Guanzhou, China

${ }^{v}$ Also at Academia Sinica Grid Computing, Institute of Physics, Academia Sinica, Taipei, Taiwan

${ }^{w}$ Also at DSM/IRFU (Institut de Recherches sur les Lois Fondamentales de l'Univers), CEA Saclay (Commissariat a l'Energie Atomique), Gif-sur-Yvette, France

${ }^{x}$ Also at Section de Physique, Université de Genève, Geneva, Switzerland

${ }^{y}$ Also at Departamento de Fisica, Universidade de Minho, Braga, Portugal

${ }^{\mathrm{z}}$ Also at Department of Physics and Astronomy, University of South Carolina, Columbia SC, United States of America

${ }^{a}$ Also at Institute for Particle and Nuclear Physics, Wigner Research Centre for Physics, Budapest, Hungary

${ }^{a b}$ Also at California Institute of Technology, Pasadena CA, United States of America

${ }^{a c}$ Also at Institute of Physics, Jagiellonian University, Krakow, Poland

${ }^{a d}$ Also at Institute of High Energy Physics, Chinese Academy of Sciences, Beijing, China 


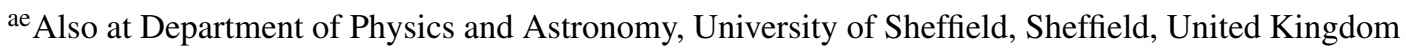

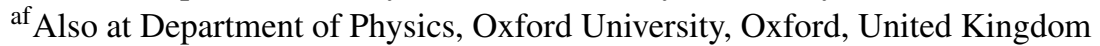

${ }^{\text {ag }}$ Also at Institute of Physics, Academia Sinica, Taipei, Taiwan

ah Also at Department of Physics, The University of Michigan, Ann Arbor MI, United States of America

${ }^{\text {ai }}$ Also at Laboratoire de Physique Nucléaire et de Hautes Energies, UPMC and Université Paris-Diderot and CNRS/IN2P3, Paris, France

*Deceased 